\title{
Structural and Photophysical Templating of Conjugated Polyelectrolytes with Single-Stranded DNA
}

Lisa Peterhans, ${ }^{\perp}$ Eliana Nicolaidou, ${ }^{\perp}$ Polydefkis Diamantis, Elisa Alloa, Mario Leclerc, Mathieu Surin, Sébastien Clément, Ursula Rothlisberger,* Natalie Banerji,* and Sophia C. Hayes*

Cite This: Chem. Mater. 2020, 32, 7347-7362

Read Online

\section{ACCESS}

山ll Metrics \& More

Article Recommendations

Supporting Information

ABSTRACT: A promising approach to influence and control the photophysical properties of conjugated polymers is directing their molecular conformation by templating. We explore here the templating effect of single-stranded DNA oligomers (ssDNAs) on cationic polythiophenes with the goal to uncover the intermolecular interactions that direct the polymer backbone conformation. We have comprehensively characterized the optical behavior and structure of the polythiophenes in conformationally distinct complexes depending on the sequence of nucleic bases and addressed the effect on the ultrafast excited-state relaxation. This, in combination with molecular dynamics simulations, allowed us a detailed atomistic-level understanding of the structure-property

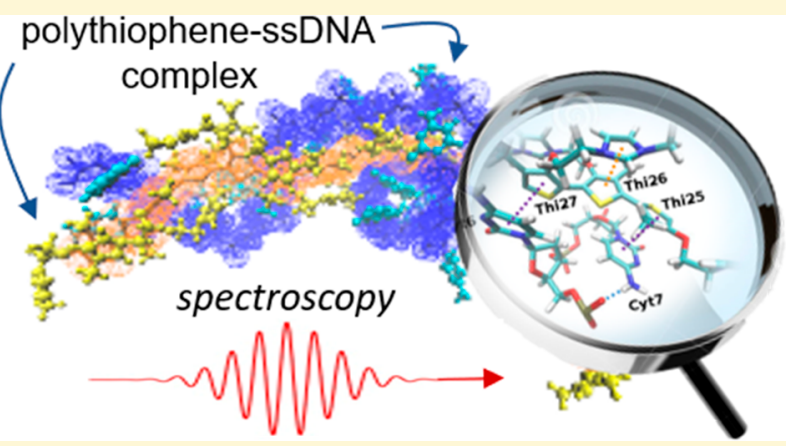
correlations. We find that electrostatic and other noncovalent interactions direct the assembly with the polymer, and we identify that optimal templating is achieved with (ideally 10-20) consecutive cytosine bases through numerous $\pi$-stacking interactions with the thiophene rings and side groups of the polymer, leading to a rigid assembly with ssDNA, with highly ordered chains and unique optical signatures. Our insights are an important step forward in an effective approach to structural templating and optoelectronic control of conjugated polymers and organic materials in general.

\section{INTRODUCTION}

Control over the conformation of functional molecules in order to tailor their optoelectronic properties has tremendous potential for technological applications, especially for organic $\pi$-conjugated polymers that have gained extensive interest over the past few decades. ${ }^{1-5}$ In particular, the strong correlation of the photophysical properties of conjugated polythiophenes to their backbone conformation has been under intensive investigation. $^{6-10}$ Achieving a highly ordered and extended backbone conformation is of considerable relevance, since intrachain coupling is in this case dominant, and important delocalization as well as coherent long-range migration of excitons and charges can be realized. ${ }^{1-15}$ Such directional energy and charge transport along the chain can be exploited in electrical components, e.g., nanowires for molecular devices, ${ }^{16-18}$ cathodes in next generation batteries, ${ }^{19}$ capacitors, ${ }^{20,21}$ nanosensors, ${ }^{22}$ nanophotonics, quantum information technologies, or artificial light-harvesting systems. ${ }^{16,23,24}$ Optically, such extended conformations in polythiophenes are characterized by a red-shifted absorption, by mirror image symmetry in the absorption and emission spectra that also show an increased ratio of the first to second vibronic peaks, and by an enhanced radiative decay rate. ${ }^{25-27}$ This behavior is related to the high intrachain delocalization and can be classified as J-aggregate-like.
Unfortunately, the random-coiled form of conjugated polythiophenes is typically more favored in solutions and thin films, limiting the desired extended $\pi$-conjugation along the backbone. ${ }^{28}$ Strategies to induce extended backbone conformations range from chemical modification of the polymer (mostly the side chains), ${ }^{17,29}$ to noncovalent approaches, such as control of the electronic coupling through processing protocols, ${ }^{27}$ dilution within an inactive solid polymer matrix (polyethylene or polypropylene), ${ }^{10}$ or supramolecular assembly with templating molecules. Templates under consideration include cyclodextrins ${ }^{30-33}$ and polysaccharides ${ }^{34}$ for the formation of rod-like inclusion complexes, as well as extending charged polythiophenes through electrostatic interactions. ${ }^{35}$ Recently, nucleic acids (NAs) have emerged as particularly advantageous in directing molecular conformation, since they can conveniently induce a broad range of higherorder architectures in chiral nanoassemblies with unique and readily controllable properties. The ability of NAs to template

Received: May 29, 2020

Revised: August 6, 2020

Published: August 7, 2020 
supramolecular, programmed, and reproducible self-assembly through a combination of noncovalent interactions has been demonstrated upon complexation with conjugated polymers, ${ }^{16,28,36,37}$ surfactants, ${ }^{38}$ proteins, ${ }^{39,40}$ nanoparticles, ${ }^{41}$ and dyes. $^{23,42-44}$ Complexation of conjugated polymers with NAs is facilitated by the presence of charged side groups on the backbone of conjugated polyelectrolytes (CPEs). The strong electrostatic interactions that develop between single stranded (ss) DNA and CPEs, together with more specific and directional supramolecular effects, allow precise manipulation of the CPE conformation. Moreover, intermolecular packing between polymer backbones is in this case removed, ${ }^{45}$ allowing access to the properties of isolated conducting polymer chains with superior ease compared to other methods. $10,32,46,47$

In the present work, we focus on complexes of ssDNA with cationic poly(1H-imidazolium,1-methyl(-3-[2-[(4-methyl-3thienyl)oxy\}-ethyl]-chloride, abbreviated here as CPT (Figure 1). Cationic polythiophenes such as CPT are successful optical

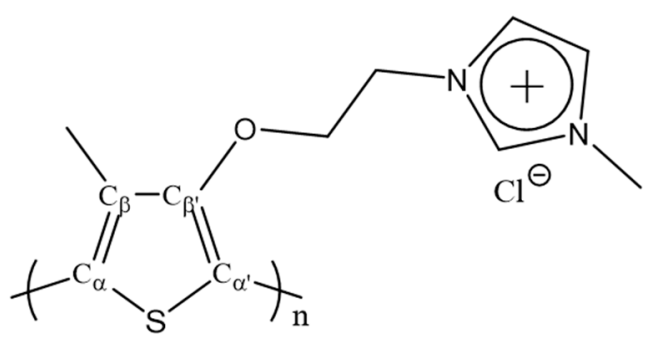

Figure 1. Molecular structure of cationic poly $(1 \mathrm{H}$-imidazolium,1methyl-3-[2-[(4-methyl-3-thienyl)oxy]-ethyl]-chloride) (CPT).

sensors of DNA chains in numerous biological assays, ${ }^{48-50}$ whereby the strong conformational changes induced in CPT upon complexation with ssDNA are the lead cause for exquisite sensitivity. ${ }^{51}$ Intriguingly, the previous biosensing work has revealed a tremendous impact of the ssDNA sequence on the conformational and optical response of the polymer. ${ }^{52}$ The complex formed of CPT and homonucleotide oligocytosine (dC) strands (Table S1) is especially noteworthy. Here, the exceptionally high ratio of the first to second vibronic peaks in the absorption spectrum points to uncommon intrachain coherence in highly ordered and extended chains. ${ }^{52}$ To fundamentally understand the atomic-level interactions that direct this unique and very desirable templating effect, we combine stationary absorption, circular dichroism (CD), femtosecond transient absorption (TA) and resonance Raman (RR) spectroscopy with resonance Raman intensity analysis (RRIA) and molecular dynamics (MD) simulations. We compare different ssDNA sequences, different lengths of $\mathrm{dC}$ strands, and various side chains on the polythiophene backbone. This establishes the structure-property correlations that link the degree of order in the backbone, the chirality of the induced conformation, and the specific interactions that develop between the cationic polythiophenes and each ssDNA strand to the optical and excited-state behavior of the complexes. We pin down key parameters leading to the strong templating effect in the $\mathrm{CPT} / \mathrm{dC}$ supramolecular assembly. On the one hand, a succession of 10-20 cytosines shows the best templating ability, as this enables numerous $\pi-\pi$ interactions between the cytosine and thiophene moieties. On the other hand, the short alkyl side chains on the polythiophene backbone facilitate further stacking interactions between the cytosines and imidazoles that stabilize the complex, while methyl substitution on $\mathrm{C}_{\beta}$ favors syn-anti conformations of $\mathrm{CPT}$, further contributing to torsional order of the polymer. Our study provides predictive understanding in an effective approach of structurally templating the optoelectronic properties of organic molecules.

\section{RESULTS AND DISCUSSION}

Optical Response of CPT/dA 20 and CPT/dC 20 . We start by considering two extreme cases where the homonucleotide ssDNA sequence containing 20 bases either strongly templates the CPT conformation $\left(\mathrm{dC}_{20}\right)$ or does not lead to significant ordering $\left(\mathrm{dA}_{20}\right)$. We correlate this to the optical properties of the complexes.

Figure 2 shows the stationary absorption and circular dichroism (CD) spectra for the two ssDNAs alone and with CPT in aqueous phosphate buffered saline (PBS) solution. The ssDNAs only absorb in the UV region, and their bands and corresponding $\mathrm{CD}$ signal are in agreement with the literature. ${ }^{53,54}$ When $\mathrm{dA}_{20}$ is mixed with $\mathrm{CPT}$ at $20{ }^{\circ} \mathrm{C}$ (Figure $2 \mathrm{a})$, an unstructured broad band appears around $510 \mathrm{~nm}$, which is ascribed to the $\pi-\pi^{*}$ transition of the polymer. ${ }^{55}$ This is different from the structured absorption band of CPT alone at $20{ }^{\circ} \mathrm{C}$ (also pictured in Figure $2 \mathrm{a}$ ), where we have previously shown significant $\mathrm{H}$-aggregation in relatively ordered chains. ${ }^{55}$ This suggests that assembly with $\mathrm{dA}_{20}$ disrupts intermolecular CPT stacking but does not support ordering of the polymer chains. Indeed, the broadness of the band indicates an ensemble of disordered backbone conformations in the complex. ${ }^{17,56}$ This is similar to the broad band at $398 \mathrm{~nm}$ of $\mathrm{CPT}$ alone at $55{ }^{\circ} \mathrm{C}$ (Figure 2a), which we have assigned to random coiled chains at high temperature. ${ }^{55}$ The strong redshift of disordered $\mathrm{CPT} / \mathrm{dA}_{20}$ compared to disordered CPT alone is likely due to local environment effects (e.g., change in polarity) caused by the interactions with ssDNA. ${ }^{57} \mathrm{CPT} / \mathrm{dA}_{20}$ at $20{ }^{\circ} \mathrm{C}$ shows a $(+/-) \mathrm{CD}$ signature with a positive band centered at $593 \mathrm{~nm}$ and a negative band at around $500 \mathrm{~nm}$ (unlike achiral CPT alone, Figure S2), meaning that the complexation with $\mathrm{dA}_{20}$ induces some chirality to the polymer with a preferential right-handed helical structure. ${ }^{53,58-63}$ This conformation is however flexible and varies with temperature. At $55{ }^{\circ} \mathrm{C}$, the absorption band blue-shifts (increase in chain disorder $)^{55}$ with no evident $\mathrm{CD}$ signature in the visible region (Figure S2). ${ }^{52}$ On the other hand, the $\mathrm{CD}$ signal due to $\mathrm{dA}_{20}$ in the UV region, indicating a right-handed helical conformation, changes only slightly upon complexation with CPT at any temperature, in agreement with the calculated CD spectrum (see Figure S6) and the UVRR results below, implying that the secondary structure of $\mathrm{dA}_{20}$ is largely unaffected by assembly with CPT, ${ }^{64}$ pointing to weak interactions in the complex.

The experimental findings are strongly supported by our $\mathrm{MD}$ simulations. Those show that the noncomplexed $\mathrm{dA}_{20}$ fragment displays almost no conformational flexibility and maintains a rigid conformation throughout the simulation, stabilized by stacking interactions between adenine bases (Figure S5a and close contact analysis below), in agreement with the literature. ${ }^{64,65}$ In the complex with CPT, two independent $\mathrm{MD}$ runs show limited specific interactions between the polymer and $\mathrm{dA}_{20}$ as well as an unstable structure of $\mathrm{CPT} / \mathrm{dA}_{20}$ (Figure $2 \mathrm{c}$ ). While CPT displays flexibility (in agreement with the broad unstructured absorption spectrum), the $\mathrm{dA}_{20}$ fragment maintains a significant degree of helicity during most of the simulation time scale $(1.54 \mu \mathrm{s})$, albeit in the form of two helices approximately comprising the first five and 

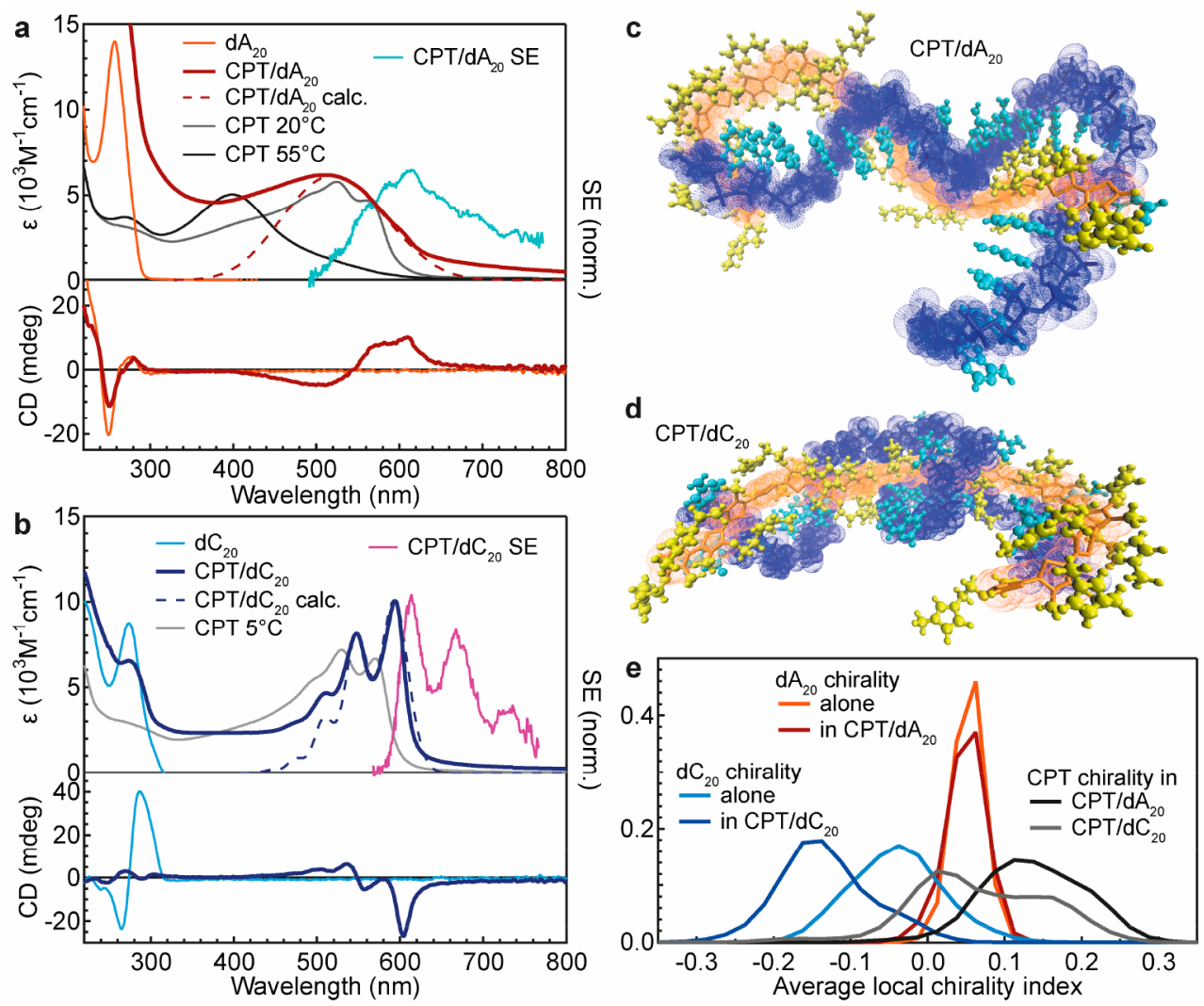

Figure 2. Experimental and calculated absorption (top left axis), stimulated emission (SE; right axis) and CD (bottom left axis) spectra of (a) CPT at 20 and $55^{\circ} \mathrm{C}, \mathrm{dA}_{20}$, and $\mathrm{CPT} / \mathrm{dA}_{20}$ at $20^{\circ} \mathrm{C}$ and (b) CPT at $5{ }^{\circ} \mathrm{C}, \mathrm{dC}_{20}$, and $\mathrm{CPT} / \mathrm{dC}_{20}$ at $20{ }^{\circ} \mathrm{C}$ (complex formed at $55^{\circ} \mathrm{C}$ ). All solutions were in PBS buffer with a concentration of $7.3 \times 10^{-5} \mathrm{M}$ (monomeric basis). For the CPT/ssDNA complexes, the monomeric equivalence between positive and negative charges was achieved. The SE spectra were obtained by subtracting the calculated absorption spectra from the transient absorption (TA) spectra at 2 ps after photoexcitation. Representative structures of (c) CPT/ $\mathrm{dA}_{20}$ and (d) CPT/dC 20 assemblies obtained by MD simulations. The ssDNA and the CPT chains are represented as follows. (i) CPT backbone: orange licorice surrounded by an orange dotted surface. (ii) CPT side chains: yellow licorice. (iii) DNA backbone (phosphate + sugar): blue licorice surrounded by a blue dotted surface. (iv) DNA bases: cyan licorice. (e) Distributions of the chirality indexes for $\mathrm{dA}_{20}$ alone and in CPT/ $\mathrm{dA}_{20}, \mathrm{dC}_{20}$ alone and in CPT/dC 20 , and CPT in $\mathrm{CPT} / \mathrm{dA}_{20}$ and in $\mathrm{CPT} / \mathrm{dC}_{20}$ obtained over the entire length of the MD simulations. A value of 0 corresponds to an achiral molecule, while the higher the absolute value of $\mathrm{CI}_{i, i+1}$, the more chiral the molecule is. Positive and negative values correspond to right-handed and left-handed orientations, respectively.

the last 15 adenines, respectively. We have further quantified the helical order and chirality in the MD simulations, using an average local chirality index $\left(\mathrm{CI}_{i, i+1}\right.$, see Computational Methods for details), similar to the one recently reported. ${ }^{53}$ The average chirality index distribution of $\mathrm{dA}_{20}$ alone is very similar to that of the same ssDNA fragment in the complex with CPT (Figure 2e), and the positive values point to a righthanded helical configuration, which, as discussed above, is maintained in $\mathrm{CPT} / \mathrm{dA}_{20}$. The distribution of the chirality index of $\mathrm{CPT}$ in $\mathrm{CPT} / \mathrm{dA}_{20}$ visits only positive values, in agreement with the $\mathrm{CD}$ signal in the CPT-absorbing region, thus confirming that a right-handed helicity is also induced in the polymer backbone.

In stark contrast to the absorption spectrum of $\mathrm{CPT} / \mathrm{dA}_{20}$, the spectrum of the $\mathrm{CPT} / \mathrm{dC}_{20}$ complex shows a red-shifted absorption, strong vibronic structure (with a spacing of 1447 $\mathrm{cm}^{-1}$ due to the symmetric $\mathrm{C}-\mathrm{C}$ and $\mathrm{C}=\mathrm{C}$ stretching of the thiophene units), and a predominant $0-0$ transition at $594 \mathrm{~nm}$ (Figure 2b), as generally observed for extended polythiophene chains with a large degree of order. ${ }^{25,26}$ Here, the $A_{0-0} / A_{0-1}$ ratio is 1.24 (for a complex formed at $55^{\circ} \mathrm{C}$ and then cooled to $20{ }^{\circ} \mathrm{C}$, and 1.16 if directly prepared at $20^{\circ} \mathrm{C}$, Figure S3). This is, to the best of our knowledge, the highest ratio observed so far, even higher than for P3HT in nanofibers. ${ }^{27}$ In addition, once formed, the $\mathrm{CPT} / \mathrm{dC}_{20}$ complex is very rigid compared to $\mathrm{CPT} / \mathrm{dA}_{20}$, as the absorption band shape remains largely unaffected by temperature variations (see Figure S3a). Moreover, an induced CD signal due to CPT in a left-handed helical conformation appears in $\mathrm{CPT} / \mathrm{dC}_{20}$ between 450 and $670 \mathrm{~nm}$ (Figure 2b), suggesting the induction of different helical structures upon complexation with each of the ssDNA chains. The trend of the experimental CD signals for CPT in the two complexes (a shift from right-handed to left-handed conformations when going from $\mathrm{CPT} / \mathrm{dA}_{20}$ to $\mathrm{CPT} / \mathrm{dC}_{20}$ ) is qualitatively reproduced by the $\mathrm{MD}$ simulations with more lefthanded conformations being sampled for CPT in CPT/dC20 compared to CPT/dA20. The distribution of the local chirality indices of CPT is broad including both positive and negative values with a large statistical error (Figure 2e). This suggests that in spite of the length of the MD simulation $(1.56 \mu \mathrm{s})$, the sampling of the local chirality index of CPT in CPT/dC20 is not yet fully converged, preventing a direct quantitative comparison of the theoretically predicted absolute CPT handedness with the experimental one. Overall, the MD simulations confirm that $\mathrm{CPT} / \mathrm{dC}_{20}$ adopts and maintains a structure that is stabilized by persistent intermolecular 

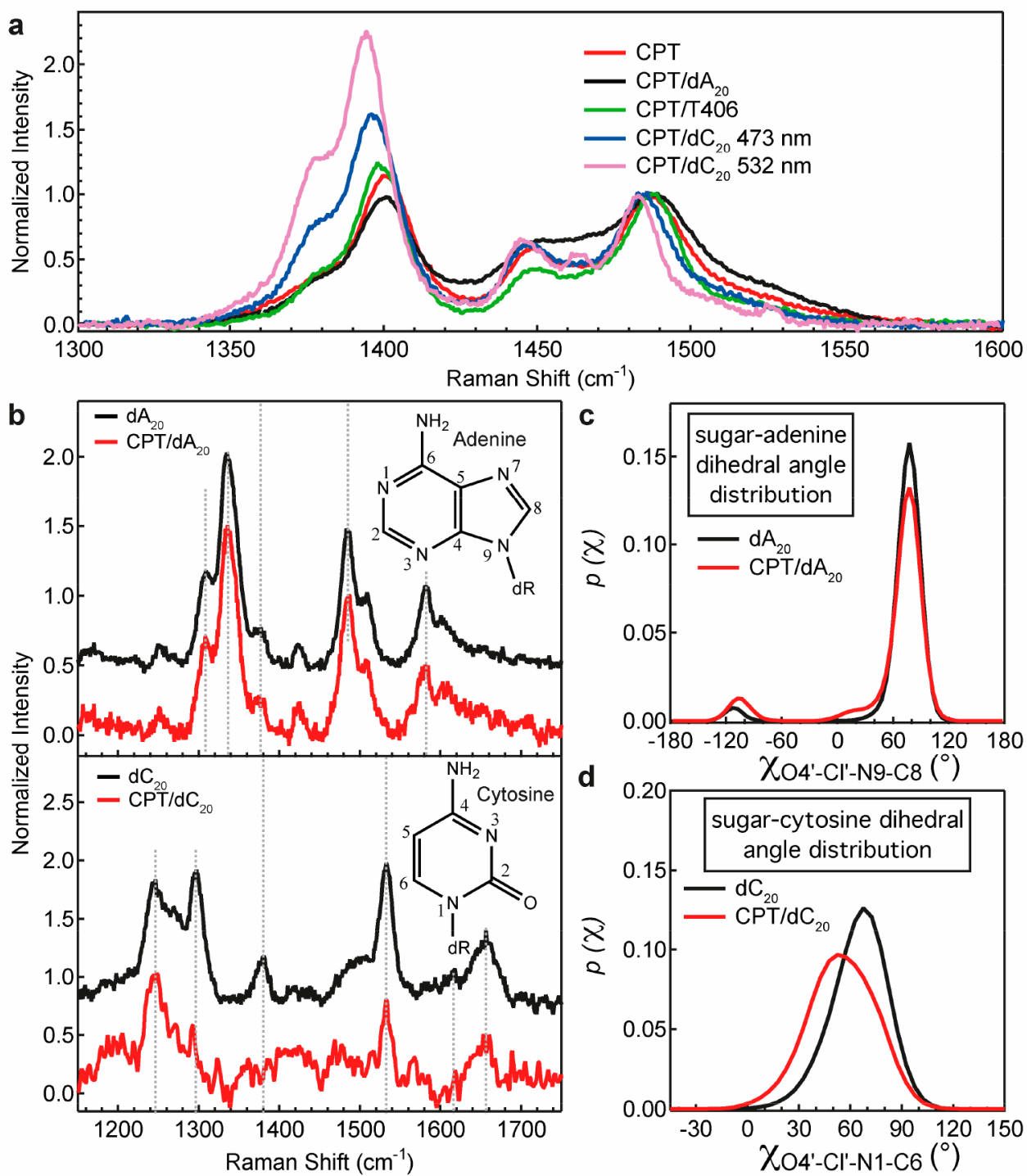

Figure 3. (a) Resonance Raman (RR) spectra with visible excitation at $473 \mathrm{~nm}$ for CPT, CPT/ $/ \mathrm{A}_{20}, \mathrm{CPT} / \mathrm{T} 406$, and $\mathrm{CPT} / \mathrm{dC} \mathrm{C}_{20}$ and at $532 \mathrm{~nm}$ for $\mathrm{CPT} / \mathrm{dC}_{20}$ in PBS buffer with a concentration of $1.5 \times 10^{-4} \mathrm{M}$ (monomeric basis) at $20{ }^{\circ} \mathrm{C}$. (b) UVRR spectra of $\mathrm{dA}_{20}$ and $\mathrm{CPT} / \mathrm{dA}_{20}$ in PBS buffer with a concentration of $1.5 \times 10^{-4} \mathrm{M}$ and $\mathrm{dC}_{20}$ and $\mathrm{CPT} / \mathrm{dC}_{20}$ in PBS buffer with a concentration of $3 \times 10^{-4} \mathrm{M}$ (monomeric basis) at room temperature with excitation at $266 \mathrm{~nm}$ (normalized with respect to the intensity of the band at $\sim 1484$ and $\sim 1246 \mathrm{~cm}^{-1}$ respectively). (c,d) Distributions of the dihedral angle between the sugar (deoxyribose) and the base in ssDNA alone and assembled with CPT obtained by the MD simulations ( (c) adenine in $\mathrm{dA}_{20}$ and $(\mathrm{d})$ cytosine in $\left.\mathrm{dC}_{20}\right)$.

interactions (see below) between the ssDNA and the polymer (Figure 2d). All this points to strong conformational templating of $\mathrm{CPT}$ by $\mathrm{dC}_{20}$, leading to extended isolated chains with predominant intrachain coupling and J-aggregatelike behavior. ${ }^{66-68}$ In comparison, the absorption spectrum of $\mathrm{CPT}$ alone at $5{ }^{\circ} \mathrm{C}$ is also pictured in Figure $2 \mathrm{~b}$, where the polymer is in an ordered conformation but shows $\mathrm{H}$ aggregation between CPT chains. ${ }^{55}$ Here, the $A_{0-0} / A_{0-1}$ ratio is only 0.93 and the $0-0$ peak is suppressed due to the interchain coupling. ${ }^{27}$

Given the strong interactions between $\mathrm{CPT}$ and $\mathrm{dC}_{20}$, the conformation of the ssDNA strand is also significantly affected in the complex. $\mathrm{dC}_{20}$ alone has a characteristic $\mathrm{CD}$ signal with a dominant positive band at $288 \mathrm{~nm}$ and a negative band at $265 \mathrm{~nm}$, suggesting the presence of a moderate portion of $\mathrm{i}$ motif structure (Figure S4), as expected for cytosine-rich DNA strands at $\mathrm{pH} 7.3$ (i-motif formation is generally more important at slightly acidic $\mathrm{pH}$, when hemiprotonation of cytosines favors the formation of hydrogen bonds between them, see SI). ${ }^{54,69,70} \mathrm{MD}$ simulations show that $\mathrm{dC}_{20}$ has a high degree of flexibility and adopts several different structures, including both extended and compact configurations (Figures $\mathrm{S} 5 \mathrm{~b} / \mathrm{c}$ ) that sample right- and left-handed orientations (Figure 2e). While compact $\mathrm{dC}_{20}$ structures are observed (e.g., Figure $\mathrm{S} 5 \mathrm{~b}$ ), which are mainly stabilized by a varying number of $\mathrm{H}$ bonds between cytosine bases, the i-motif structure is not evidenced by MD simulations since the simulations were performed with fixed protonation states. Nevertheless, the mixture of secondary structures is theoretically demonstrated as well. This is in stark contrast to the more rigid $\mathrm{dA}_{20}$ and likely favors the assembly of $\mathrm{dC}_{20}$ with CPT. In the complex, the $\mathrm{CD}$ feature of $\mathrm{dC}_{20}$ is dramatically changed (Figure $2 \mathrm{c}$ ), in agreement with the reduced ellipticity in the calculated CD spectrum (Figure S6), indicating that the secondary structure 
of the ssDNA strand is strongly modified upon complexation with CPT (see also UVRR results below). The absorption peak at $274 \mathrm{~nm}$ is also less intense in the complex, possibly due to hypochromism induced by a partial alignment of the transition dipole moments when the bases are stacked. ${ }^{71}$ This phenomenon is known for double stranded DNA, which absorbs less compared to the denatured form. ${ }^{72}$ The MD simulations confirm that the chirality distribution of $\mathrm{dC}_{20}$ in $\mathrm{CPT} / \mathrm{dC}_{20}$ is fully shifted to negative values (left-handed orientations) of the average $\mathrm{CI}_{i, i+1}$, sampling larger absolute values of the average local chirality indexes compared to $\mathrm{dA}_{20}$ in $\mathrm{CPT} / \mathrm{dA}_{20}$.

Backbone Planarity of CPT in the Complexes with $\mathbf{d A}_{20}$ and $\mathbf{d C}_{20}$. A more in-depth experimental investigation of the backbone planarity of CPT upon complexation with the different ssDNA chains was achieved by using resonance Raman (RR) spectroscopy. This confirms the high degree of intrachain order in $\mathrm{CPT} / \mathrm{dC}_{20}$.

Figure 3a presents the fingerprint region of the RR spectra of CPT complexes with visible excitation (for the extended range spectra, see Figure S7), which were normalized with respect to the intensity of the band at $\sim 1487 \mathrm{~cm}^{-1}$. At the used wavelengths (473 and $532 \mathrm{~nm}$ ), we are on resonance with the $\pi-\pi^{*}$ absorption of CPT, therefore we observe only contributions associated with vibrations of the conjugated polymer (mainly the thiophene backbone). The spectra are similar to the RR spectra of CPT that we have reported before and assigned based on DFT calculations. ${ }^{55}$ The deconvolution of the RR spectra is shown in Figure S8, and the band assignment is summarized in Table S5. The position, intensity, and line width of the Raman bands are strongly dependent on the ssDNA participating in the complex. In the case of CPT/ $\mathrm{dA}_{20}$, the bands appear essentially at the same position as in the case of the polymer alone, while a $3-4 \mathrm{~cm}^{-1}$ downshift is observed in the case of $\mathrm{CPT} / \mathrm{dC}_{20}$. Moreover, the relative intensities of the $\mathrm{C}-\mathrm{C}\left(\sim 1400 \mathrm{~cm}^{-1}\right)$ and $\mathrm{C}=\mathrm{C}\left(1487 \mathrm{~cm}^{-1}\right)$ stretches in $\mathrm{CPT} / \mathrm{dC}_{20}$ are distinctly different than in the other complexes or the polymer alone, with the $\mathrm{C}-\mathrm{C}$ intensity dominating. Taken in conjunction with the downshifts in the bands, this signifies an increase in the electronic conjugation of the CPT backbone caused by a greater inter-ring planarity, demonstrating a more extended conformation for CPT in the $\mathrm{CPT} / \mathrm{dC}_{20}$ complex. ${ }^{55}$ The intensity of the $\mathrm{C}-\mathrm{C}$ band increases further with excitation at $532 \mathrm{~nm}$, due to resonance with even more planar and lower energy chain segments. In addition, the spectrum of $\mathrm{CPT} / \mathrm{dA}_{20}$ exhibits broader line widths, showing inhomogeneity and torsional disorder of the $\mathrm{CPT}$ conformation. In contrast, the narrow peaks observed in the $\mathrm{CPT} / \mathrm{dC}_{20}$ spectrum reflect the rigidity and homogeneity of the CPT conformation in the complex.

With the help of MD simulations, the distribution of the S$\mathrm{C}-\mathrm{C}-\mathrm{S}$ dihedral angles (between adjacent thiophene rings) was calculated for CPT alone and compared to the corresponding values in the two complexes (Figure $S 9$ in SI). Overall, the occurrence of a dihedral angle corresponding to a planar conformation (around $0^{\circ},-180^{\circ}$ or $+180^{\circ}$ ) follows the trend $\mathrm{CPT} / \mathrm{dC}_{20} \gg \mathrm{CPT} / \mathrm{dA}_{20}>\mathrm{CPT}$ alone, which agrees with the increased degree of planarity for $\mathrm{CPT} / \mathrm{dC}_{20}$ witnessed by RR spectroscopy with visible excitation.

Atomistic-Level Details of the CPT-ssDNA Interactions. We have shown that $\mathrm{dA}_{20}$ does not induce strong conformational changes in CPT, while $\mathrm{dC}_{20}$ leads to a tighter structure for the $\mathrm{CPT} / \mathrm{dC}_{20}$ complex, with pronounced optical signatures of the polymer. To understand this selective response to different ssDNA bases, we have investigated the atomistic-level interactions in the complexes during the MD simulations using a close contact analysis. We evaluate (1) electrostatic interactions between the negatively charged phosphate groups of the ssDNA and the positively charged imidazole side-chains of CPT, (2) various types of $\pi$-stacking (between the cytosine/adenine bases and the thiophenes or imidazoles of CPT, intra-DNA $\pi$-stacking between the nucleobases, intra-CPT $\pi$-stacking between the thiophene and imidazole rings), and (3) intra-DNA and CPT/DNA Hbonding (between the $\mathrm{NH}_{2}$ groups of the nucleobases and the oxygen of the CPT thiophenes). This is then experimentally confirmed by RR spectroscopy in the UV (266 nm), which interrogates structural changes that occur in the various ssDNAs upon complexation with CPT.

We find that electrostatic interactions are crucial to bringing the $\mathrm{CPT}$ and ssDNA strands together and play a significant role in both the $\mathrm{CPT} / \mathrm{dA}_{20}$ and $\mathrm{CPT} / \mathrm{dC}_{20}$ complexes (Figures S11-S14). This agrees with the efficient assembly of CPT with both ssDNA strands, evidenced by the stationary absorption and $C D$ spectra, as well as via the reduced excited-state lifetimes of both complexes (see below). In $\mathrm{CPT} / \mathrm{dA}_{20}$, the pronounced intra-DNA $\pi$-stacking (between neighboring adenine bases, Figure S15.3) and thiophene-imidazole interactions within CPT (Figure S15.5) prevail over the stacking between the ssDNA and CPT units (adeninethiophene/imidazole, Figure S15.1 and S15.2), leading to conformational flexibility and broad absorption of CPT in the complex. The $\mathrm{MD}$ simulations also show that $\mathrm{CPT} / \mathrm{dA}_{20}$ displays limited intra-DNA $\mathrm{H}$-bonding interactions between $\mathrm{NH}_{2}$ groups of adenine bases and phosphate groups, and a similar number of weak CPT-DNA H-bonds (Figures S17 and $\mathrm{S} 18 \mathrm{a})$. Thus, electrostatic interactions are principally responsible for the overall structure of $\mathrm{CPT} / \mathrm{dA}_{20}$. The weak adenineCPT stacking is experimentally confirmed by the UVRR analysis. Figure $3 \mathrm{~b}$ presents the UVRR spectra of each ssDNA alone and in the complex (extended spectral range in Figure S10). The spectra of the ssDNAs are similar to those previously reported (see Table S6 for the assignment of the main bands). ${ }^{73-76}$ Characteristic bands of ssDNA are associated with in-plane vibrational modes of the base rings and exocyclic bonds. Sugar and phosphate vibrations are not enhanced at this excitation wavelength and are therefore not identified in the UVRR spectra. As reported in the past, ${ }^{65}$ the vibrational bands in the UVRR spectra of NAs are highly sensitive to structural changes. Thus, the great similarity displayed between the Raman spectra of $\mathrm{dA}_{20}$ and CPT/ $\mathrm{dA}_{20}$ reflects the resistance to deformation due to the intrinsic rigidity of single-stranded $\mathrm{dA}_{20}$, which is stabilized by the stacking interactions between adenine bases. ${ }^{64,65}$

In contrast, the Raman bands at 991, 1297, 1381, and 1532 $\mathrm{cm}^{-1}$, which correspond to vibrational modes of cytosine localized near the glycosidic bond, experience an intensity decrease when $\mathrm{dC}_{20}$ assembles with CPT (Figures $3 \mathrm{~b}$ and S10). Previous work has shown that those base vibrations are affected by the deoxyribose ring puckering and the glycosidic bond orientation defined by the torsion angle $(\chi) .{ }^{65,77-79}$ The conformational sensitivity of the in-plane base vibrations derives from vibrational coupling with the adjacent $\mathrm{C}\left(\mathrm{1}^{\prime}\right)-\mathrm{H}$ bend from the deoxyribose ring (see Figure S10e). Therefore, the changes observed in peak amplitude are likely associated with a transition in sugar puckering and alteration of the 
torsional angle between the cytosine and the deoxyribose, induced by specific interactions between $\mathrm{dC}_{20}$ and CPT that are absent in $\mathrm{CPT} / \mathrm{dA}_{20}$. Indeed, according to our $\mathrm{MD}$ analysis, the distribution of dihedral angles between the base and the sugar for $\mathrm{dC}_{20}$ alone and assembled with CPT shifts by $20^{\circ}$ (Figure 3d), while no such difference is seen for the sugarbase dihedral angle of $\mathrm{dA}_{20}$ (Figure 3c). Moreover, the MD close contact analysis shows that strong $\pi$-stacking develops between the cytosines of $\mathrm{dC}_{20}$ and the thiophenes and imidazoles of CPT (Figure S15.1 and S15.2). These $\pi$-stacking interactions are responsible for the modification in the deoxyribose ring puckering and/or twist of the glycosidic bond. They are also the main reason for the important differences observed between the CPT/ $\mathrm{dC}_{20}$ and $\mathrm{CPT} / \mathrm{dA}_{20}$ complexes. Characteristically, five cytosines stack against a thiophene ring in $\mathrm{CPT} / \mathrm{dC}_{20}$ during more than $50 \%$ of the total simulation time, and two more cytosines for more than $40 \%$ of the time (Figure S15.1). In contrast, only three adenines stack against a thiophene for more than $40 \%$ of the total $\mathrm{MD}$ time in $\mathrm{CPT} / \mathrm{dA}_{20}$. As depicted in Figure 4, the

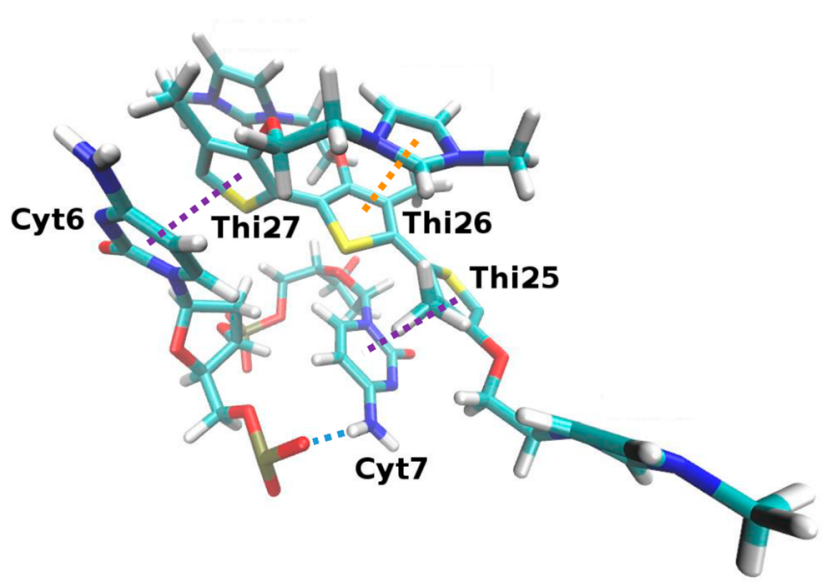

Figure 4. Image of noncovalent interactions occurring between CPT and $\mathrm{dC}_{20}$ as observed in $\mathrm{MD}$ simulations. $\pi$-stacking interactions between cytosine and thiophene rings are shown in purple dotted lines, while stacking interactions between the imidazole group from a neighboring thiophene and a thiophene ring are shown in orange. A hydrogen bond between the $\mathrm{N}-\mathrm{H}$ proton from the cytosine and the oxygen from a phosphate group is also apparent in this snapshot (cyan dotted line).

cytosines stack with every other thiophene in the sequence, due to the long distance between bases imposed by the phosphodiester bond. The close contact analysis also shows that nucleobase-imidazole interactions in $\mathrm{CPT} / \mathrm{dC}_{20}$ are much more abundant than in $\mathrm{CPT} / \mathrm{dA}_{20}$ (Figure S15.2). Both $\pi-\pi$ interactions thus play a decisive role in defining the compact structure of $\mathrm{CPT} / \mathrm{dC}_{20}$ and are further complemented by thiophene-imidazole stacking within the CPT backbone (Figures 4 and S15.5).

Intra-DNA cytosine stacking in $\mathrm{dC}_{20}$ is not as strong as the adenine interactions in $\mathrm{dA}_{20}$ (Figures S15.3 and S15.4), ${ }^{80,81}$ rendering the cytosines available for $\pi$-stacking with the thiophenes and imidazoles from CPT. Moreover, the MD close contact analysis demonstrates a significant decrease in cytosine stacking once CPT is added (Figure S15.4). Any secondary structure that $\mathrm{dC}_{20}$ can adopt on its own is thus disrupted, and the ssDNA chain is forced into a different conformation during complex formation as attested by UVRR and the CD signature in the UV (see Figures $2 \mathrm{c}$ and $3 \mathrm{~b}$ ). Finally, the local structure of the $\mathrm{CPT} / \mathrm{dC}_{20}$ complex is stabilized by $\mathrm{H}$ bonds within the $\mathrm{dC}_{20}$ strand, mainly between amine groups of cytosine and oxygen atoms of phosphate, which are far more abundant than weak $\mathrm{H}$ bonds between DNA and CPT (Figure S18b). Thus, $\mathrm{H}$-bonding, rather than stacking, predominantly determines the conformation of $\mathrm{dC}_{20}$ in the assembly, in line with the loss of intra-DNA stacking once CPT is added (Figure S15.4). The $\mathrm{MD}$ simulations indicate that these intra-DNA H-bonding interactions commonly occur in conjunction with cytosinethiophene stacking interactions, as depicted in Figure 4, contributing to the overall conformational stability of CPT/ $\mathrm{dC}_{20}$ (more pictures of noncovalent interactions can be found in Figures S14 and S17). On the other hand, chalcogen bonds within CPT, between the sulfur atoms of the thiophenes and the oxygen atoms of the alkoxy side chains, ${ }^{82}$ are found to have a negligible effect (see detailed discussion in the SI, section S5.5).

Effect of dC Length on CPT/dC Complex Formation. Since the cytosines of the ssDNA strand stack with every second thiophene of CPT (Figure 4), this suggests that consecutive sequences of cytosines favor maximal $\pi$-stacking occurrence and chain extension. We thus investigate what length of $\mathrm{dC}$ induces the best templating of CPT, which consists of an average of 42 thiophene units (estimated using the number-averaged molecular weight, $\mathrm{Mn}$ ).

Figure 5a shows the absorption and CD spectra of CPT complexed to dC strands of 5 to 80 bases, obtained by gradually adding the ssDNA to a solution of CPT in PBS buffer until a $1: 1$ ratio of thiophenes to nucleobases (monomeric equivalence) is achieved. For ssDNA oligomers with 10 units or more, the strong templating effect is always observed, yielding the characteristically structured absorption and CD spectra of CPT. Templating is typically complete at a thiophene/nucleobase ratio of 1:0.75-1 (maximal $A_{0-0} / A_{0-1}$ ratio, see Figure $S 20$ for the titration results). However, the $A_{0-0} / A_{0-1}$ ratio decreases as the ssDNA length increases (inset of Figure 5a, Table S7). Optimal templating resulting in the most ordered and extended CPT conformation is achieved with $\mathrm{dC}_{10}\left(A_{0-0} / A_{0-1}=1.19\right)$. This ssDNA strand is about 4 times shorter (in terms of number of monomers) than an average CPT chain. We also note that excellent templating with $\mathrm{dC}_{10}$ already occurs at half the monomeric equivalence (Figure S20b), in line with the fact that the cytosines stack with every second thiophene of CPT. We suggest that (two to four) 10 nucleobase units are ideal to wrap around segments of CPT forming a compact structure via mainly electrostatic and $\pi$-stacking interactions as shown in Figure $2 \mathrm{~d}$, leading to significant local ordering and intrachain coherence. For longer ssDNA segments (especially $>20$ bases), the assembly over the entire length of the two chains is more challenging and might compete with increased intra-DNA interactions. Noteworthy is the similar shape of the UV-CD signature for $\mathrm{dC}_{80}$ alone and $\mathrm{CPT} / \mathrm{dC}_{80}$ (Figure S21), showing that complex formation leads in this case to a smaller change in the ssDNA conformation, ultimately explaining the lower templating effect in this complex $\left(A_{0-0} / A_{0-1}=1.06\right)$.

Finally, we find that complex formation is not complete for one monomeric equivalent of $\mathrm{dC}_{5}$, since only a slight effect on the polymer absorption and $C D$ signal is observed, and a shoulder around $400 \mathrm{~nm}$ due to disordered CPT chains remains visible (Figure 5a). To achieve significant templating with $\mathrm{dC}_{5}$, an important excess of ssDNA needs to be added 

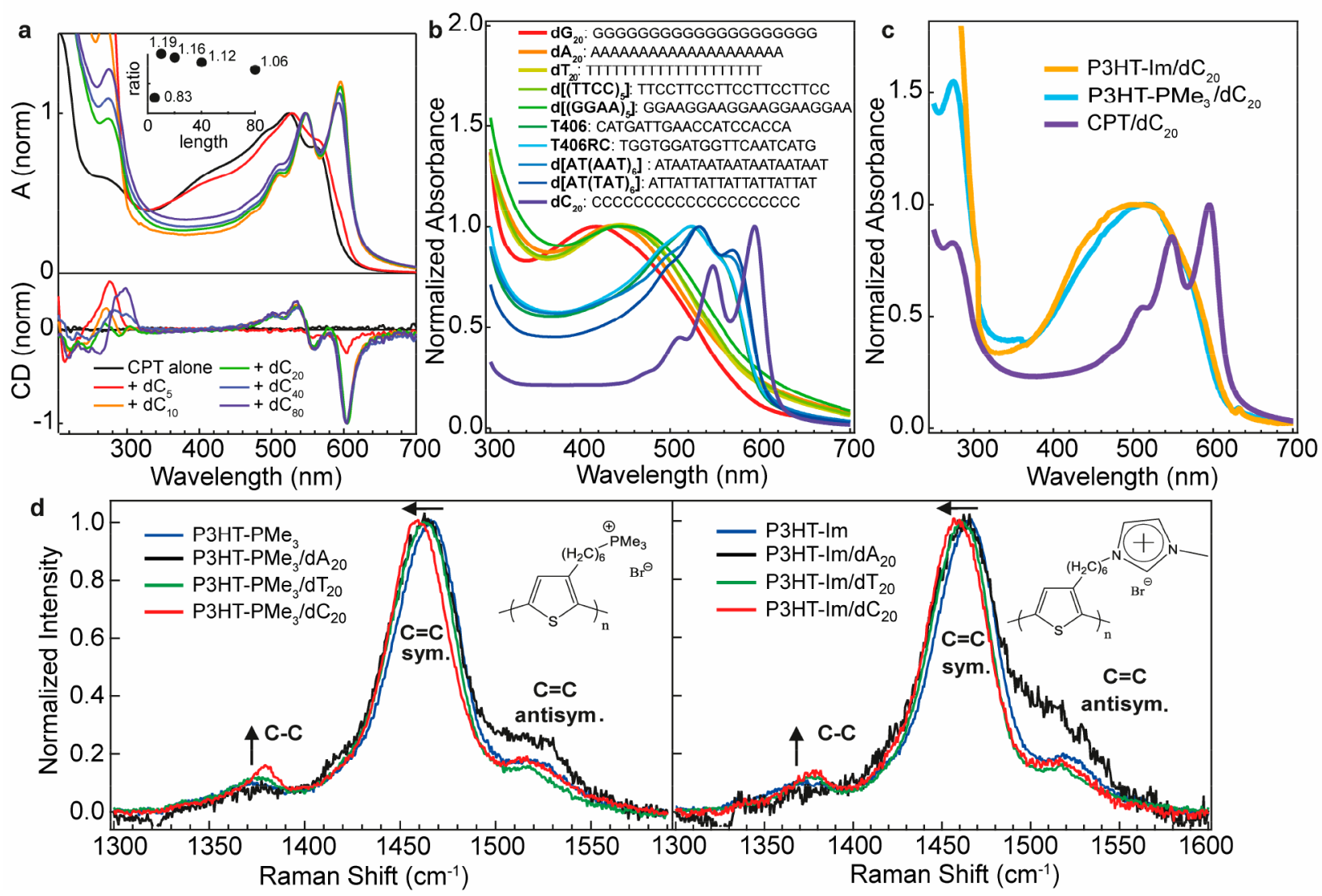

Figure 5. (a) Stationary absorption and CD spectra of CPT complexed to $\mathrm{dC}_{20}$ strands of 5, 10, 20, 40, and 80 nucleobase units at $20{ }^{\circ} \mathrm{C}$ (precipitation occurred for the longer ssDNA strands at $55^{\circ} \mathrm{C}$ ), obtained by adding monomeric equivalence of the ssDNAs to a solution of CPT in PBS buffer $\left(7.3 \times 10^{-5} \mathrm{M}\right.$, monomeric basis). The inset shows the $A_{0-0} / A_{0-1}$ ratio of the CPT absorption band as a function of $\mathrm{dC}_{20}$ length $(1: 1$ monomeric equivalence, $20{ }^{\circ} \mathrm{C}, 10 \mathrm{~min}$ stirring of the solution). (b) Absorption and CD spectra of CPT complexed to various 20-base ssDNA strands (sequences shown in the legend) at $55{ }^{\circ} \mathrm{C}$. (c) Absorption spectra of $\mathrm{dC}_{20}$ complexed to CPT, P3HT-PMe 3 , and P3HT-Im in PBS buffer with a concentration of $1.5 \times 10^{-4} \mathrm{M}$ (monomeric basis) at room temperature. (d) RR spectra normalized with respect to the intensity of the band at $\sim 1460 \mathrm{~cm}^{-1}$ with excitation at $473 \mathrm{~nm}$ for (left) P3HT-PMe 3 or (right) P3HT-Im (blue) and its complexes with $\mathrm{dA}_{20}$ (black), dT 20 (green), and $\mathrm{dC}_{20}$ (red) in PBS buffer with a concentration of $1.5 \times 10^{-4} \mathrm{M}$ (monomeric basis) at room temperature.

(2.25 cytosine monomers for 1 thiophene unit, Figure S20). Leaving this solution for 1 day then leads to a narrow vibronic absorption structure with $A_{0-0} / A_{0-1}=1.27$ (Figure S23). We conclude that five cytosine units can already induce significant local intrachain order in CPT, involving a sequence of about 10 thiophenes. However, complexation is in this case slow and unfavorable (requiring excess ssDNA) due to modified cooperativity (see Figure S23). Titrating concentrated CPT into a dilute $\mathrm{dC}_{5}$ solution (instead of vice versa) does not lead to any templating (while the titration order is irrelevant for the longer $\mathrm{dC}$ chains), highlighting the inability of the short $\mathrm{dC}_{5}$ chains to disrupt CPT aggregates in the concentrated stock solution used for the titration (Figure S24).

Generalization to Other ssDNA and Polythiophene Systems. Having understood the specific interactions that induce characteristic conformations in complexes of CPT with $\mathrm{dA}_{20}$ and $\mathrm{dC}_{20}$, we now generalize our findings to different homonuclear and mixed ssDNA sequences, as well as to thiophene polyelectrolytes carrying different side chains. We confirm that the $\mathrm{CPT} / \mathrm{dC}_{20}$ combination is the most effective in templating an extended polymer conformation.

The absorption spectra of CPT complexed to different ssDNA sequences (20-base length, $55{ }^{\circ} \mathrm{C}$ for best complex formation) are shown in Figure $5 \mathrm{~b}$. The homonucleotides $\mathrm{dT}_{20}$ and $\mathrm{dG}_{20}$ cause weak templating similar to $\mathrm{dA}_{20}$, while $\mathrm{dC}_{20}$ stands out from all other investigated sequences. The behavior of $\mathrm{dT}_{20}$ agrees with previous work and with our $\mathrm{RR}$ measurements (Figure S7), which indicate that thymine bases do not stack strongly with thiophenes, possibly due to the extra methyl group compared to cytosine. ${ }^{53}$ The templating strength of mixed ssDNA oligomers (Table S1) is highly variable and does not necessarily correlate with the number of cytosines in the sequence. For example, the assembly of CPT with $\mathrm{d}\left[(\text { TTCC })_{5}\right]$ (50\% cytosine bases) leads to unstructured and blue-shifted absorption of CPT, possibly due to the weak stacking of the intermittent cytosine pairs with thiophenes, or due to strong intra-DNA interactions. This confirms that the excellent templating of $\mathrm{dC}_{20}$ via extended cytosine--thiophene stacking relies on consecutive cytosine sequences, not just pairs interspersed between other bases. Nevertheless, for biologically more relevant sequences containing the four natural DNA bases in random order (T406 and T406RC, with $30 \%$ and $10 \%$ cytosines, respectively), ${ }^{52}$ an intermediate templating effect can be achieved, with an optical response between those of $\mathrm{CPT} / \mathrm{dA}_{20}$ and $\mathrm{CPT} / \mathrm{dC}_{20}$ (Figure $5 \mathrm{~b})$. For $\mathrm{CPT} / \mathrm{T} 406$, the $(+/-) \mathrm{CD}$ signal at $520 \mathrm{~nm}$ indicates an induced right-handed helicity of CPT that is quite stable with temperature (Figure S26). Together with the visible RR result in Figure 3a, we thus find that the polymer adopts a more ordered conformation in the relatively rigid complex with 

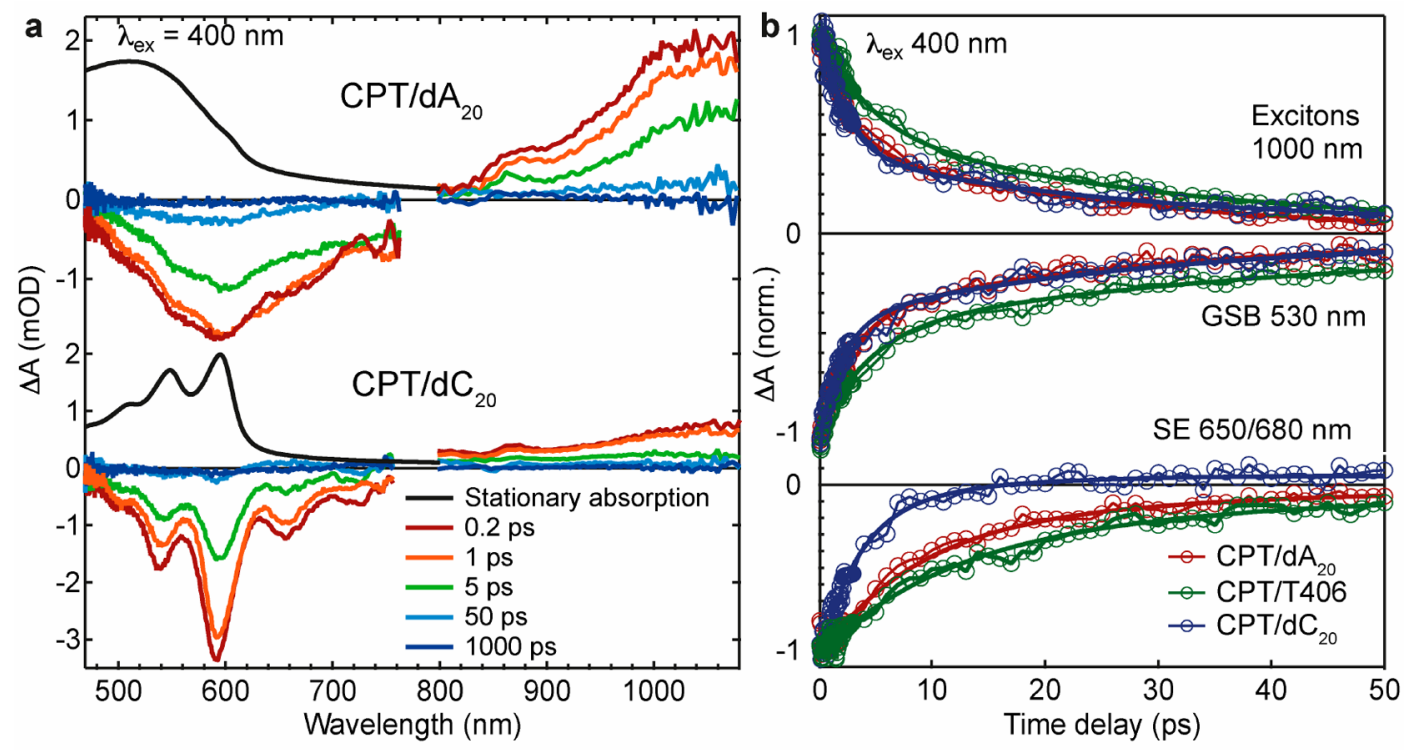

Figure 6. (a) TA spectra recorded following excitation at $400 \mathrm{~nm}$ at selected time delays with the corresponding stationary absorption (black curves) for two complexes (top, CPT/ $\mathrm{dA}_{20}$; bottom, CPT/ $\left.\mathrm{dC}_{20}\right)$ in solution at $20{ }^{\circ} \mathrm{C}\left(1.5 \times 10^{-4} \mathrm{M}\right.$ on a monomeric unit basis in PBS). The features at around 740 and $860 \mathrm{~nm}$ are artifacts due to the white light generation near the fundamental $800 \mathrm{~nm}$ laser output. (b) TA dynamics at selected probe wavelengths for the three complexes (A, T406, and C). Solid lines correspond to the biexponential global fit. $\lambda_{\mathrm{ex}}=400 \mathrm{~nm}$.

$\mathrm{T} 406$ but does not reach the level of $\mathrm{CPT} / \mathrm{dC}_{20}$. From the $\mathrm{CD}$ response of T406 in the UV range and the UVRR spectra (Figures S10 and S25), we also conclude that the ssDNA structure in the complex is not strongly modified compared to T406 alone. Interestingly, d $\left[\left(\mathrm{AT}(\mathrm{TAT})_{6}\right]\right.$ and $\mathrm{d}\left[\mathrm{AT}(\mathrm{AAT})_{6}\right]$, that contain adenines separated by thymines (but no cytosines), result in a more structured absorption spectrum than T406 when complexed with CPT. We suggest that the adenines $\pi$-stack in this case with $\mathrm{CPT}$, since our MD simulations evidence some adenine-thiophene stacking in $\mathrm{CPT} / \mathrm{dA}_{20}$ (Figure S15.1), which competes with strong interactions between the adenine bases. Disruption of the adenine-adenine stacking by the thymine bases in $\mathrm{d}[$ (AT$\left.(\mathrm{TAT})_{6}\right]$ and $\mathrm{d}\left[\mathrm{AT}(\mathrm{AAT})_{6}\right]$ can therefore explain the better complexation with CPT. However, having guanine bases between adenine pairs $\left(\mathrm{d}\left[(\mathrm{GGAA})_{5}\right]\right)$ does not lead to the same effect, showing the intricate interplay of ssDNA-CPT and intra-DNA interactions that leads to efficient conformational templating in the complexes.

With the aim to examine whether the interactions responsible for templating in the $\mathrm{CPT} / \mathrm{dC}_{20}$ system can extend to other thiophene polyelectrolytes, we chose two other polymers that were previously studied in DNA complexes $^{53,58,83,84}$ (Figure 5c). P3HT-Im and P3HT-PMe ${ }_{3}$ have different cationic side groups (phosphonium and imidazole, Figure $5 \mathrm{~d}$ ), which are attached to the $\mathrm{C}_{\beta^{\prime}}$ position of the thiophene backbone via a six-carbon chain (without the oxygen atom present in CPT). Moreover, the methyl group from the $\mathrm{C}_{\beta}$ position is missing. Even though the counterion in these polymers is bromide, its concentration is 4 orders of magnitude lower than the chloride concentration in the PBS buffer, and therefore was not considered to affect the conformation of the polymer. The RR spectra of these two polymers resemble the well-known P3HT Raman spectrum, ${ }^{27,85}$ but differ from the one of CPT, where the $\mathrm{C}=\mathrm{C}$ stretching mode is split due to alkoxy substitution at the $\mathrm{C}_{\beta^{\prime}}$ position. ${ }^{86,87}$ According to the literature, a more prominent peak around $1378 \mathrm{~cm}^{-1}(\mathrm{C}-\mathrm{C}$ stretching mode), a red-shift of the $\mathrm{C}=\mathrm{C}$ symmetric stretching band toward $\sim 1450 \mathrm{~cm}^{-1}$, a reduction of the full-width-halfmaximum (fwhm) of this $\mathrm{C}=\mathrm{C}$ mode and a less prominent peak at $\sim 1520 \mathrm{~cm}^{-1}$ (antisymmetric stretching mode of $\mathrm{C}=$ C) are all indications for a more planar P3HT backbone. These characteristic changes are most pronounced for both complexes of P3HT-PMe $\mathrm{P}_{3}$ and P3HT-Im with $\mathrm{dC}_{20}$, while $\mathrm{dA}_{20}$ has the opposite effect on the conformation of each polymer (broad RR bands, less intense $\mathrm{C}-\mathrm{C}$ peak, $\mathrm{C}=\mathrm{C}$ mode shifted to higher wavenumbers, Figures $5 \mathrm{~d}$ and S28). However, even though the effect of the ssDNA sequence on the conformation of the polymer is similar across a variety of cationic polythiophenes, with $\mathrm{dC}_{20}$ having the largest tendency to enhance backbone torsional order, its templating ability is significantly reduced with P3HT-Im and P3HT-PMe (see $^{2}$ absorption spectra, Figures $5 \mathrm{c}$ and S27). Their weak templating must result either from the longer alkyl group in the cationic side chain and/or the absence of the $\mathrm{C}_{\beta}$ methyl substituent, since our MD simulations show that chalcogen interactions (involving the alkoxy oxygen) have no decisive effect on the CPT conformation (section S5.5). Indeed, the methyl substituent in CPT restricts the possibility of cisoid (syn-syn) conformations between neighboring thiophenes, which were found quite preponderant for the interactions of P3HT-PMe 3 with ssDNA or dsDNA. ${ }^{53,83}$ Such cisoid conformations contribute to steric repulsion between the side chains inducing overall more torsional backbone disorder. Moreover, the longer alkyl chains occupy a larger volume than in CPT, possibly limiting the essential polymer-DNA $\pi$ stacking in the complexes. A systematic study of CPT side chains is under way to explore in detail the structural characteristics of the side chains necessary to form wellordered polymer chains.

Excited State Relaxation of CPT/ssDNA Complexes. In the previous sections, we have addressed the ground state conformation of polymer/ssDNA complexes. However, it is imperative to understand the impact of templating on the 

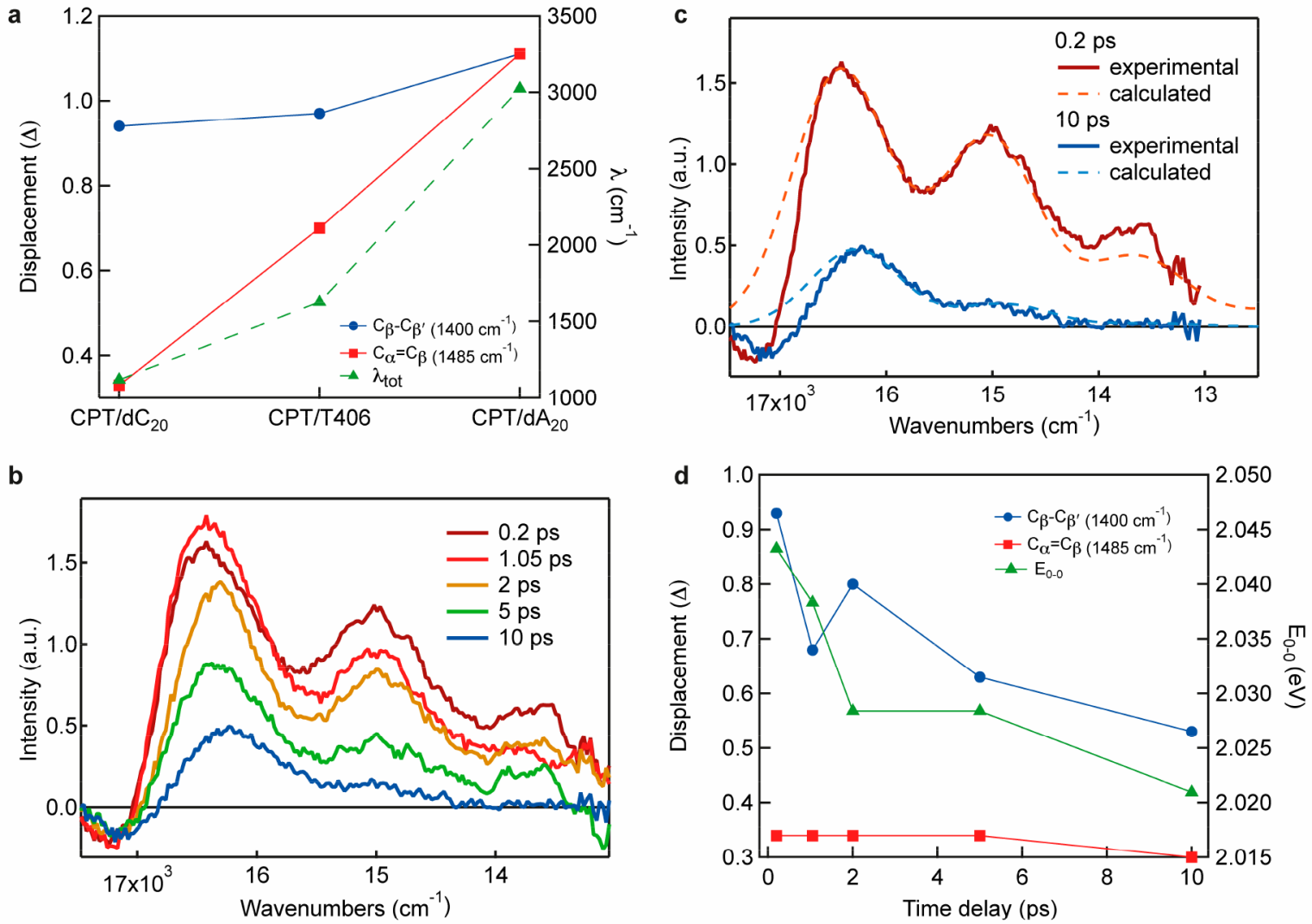

Figure 7. (a) Dependence of the displacement $(\Delta)$ along the $\mathrm{C}_{\beta}-\mathrm{C}_{\beta^{\prime}}$ and $\mathrm{C}_{\alpha}=\mathrm{C}_{\beta}$ normal coordinates on the CPT/ssDNA complex, as obtained from modeling the absorption and Raman cross sections. (b) Extracted stimulated emission spectra at different time delays after photoexcitation for $\mathrm{CPT} / \mathrm{dC}_{20}$. (c) Experimental and calculated SE spectra at 0.2 and $10 \mathrm{ps}$ after photoexcitation for $\mathrm{CPT} / \mathrm{dC} \mathrm{C}_{20}$. (d) Dependence of the displacement $(\Delta)$ along the $\mathrm{C}_{\beta}-\mathrm{C}_{\beta^{\prime}}$ and $\mathrm{C}_{\alpha}=\mathrm{C}_{\beta}$ normal coordinates on the delay time, as obtained from modeling the stimulated emission spectra.

excited state behavior, especially for the extended CPT conformation in the complex with $\mathrm{dC}_{20}$, to assess the suitability for technological applications involving e.g. extended intrachain exciton delocalization or directional long-range exciton migration. Here, we combine resonance Raman intensity analysis (RRIA) and transient absorption (TA) spectroscopy. RRIA offers a glimpse of the excited state structural evolution in the Franck-Condon region, as the band intensities are associated with specific geometrical changes upon excitation, ${ }^{88}$ while TA provides crucial insights on the lifetime and nature of the excited state.

Interestingly, even though the ssDNA sequences induce different CPT conformations, the nature and lifetime of the excited state are roughly similar in the different complexes. Figure 6 shows a selection of TA spectra for the CPT/ $\mathrm{dA}_{20}$ and $\mathrm{CPT} / \mathrm{dC}_{20}$ complexes at different time delays following excitation at $400 \mathrm{~nm}$ (CPT/T406 in Figure S29, $580 \mathrm{~nm}$ excitation in Figure S30, and global analysis in Figures S31 and S32). For all complexes, a positive broad band centered at $\sim 1050 \mathrm{~nm}$ is ascribed to the excited state absorption (ESA) of the $S_{1}$ singlet exciton, ${ }^{55}$ and a negative feature below $800 \mathrm{~nm}$ is attributed to ground state bleaching (GSB) and stimulated emission (SE), with the differences in band shape associated with different degrees of order in CPT as also reflected in the absorption spectra. The broad and red-shifted ESA is characteristic of delocalized excitons (as we observed for ordered CPT alone at low temperature, ${ }^{55}$ while the ESA of more localized excitons in random-coiled CPT chains is narrower and centered around $950 \mathrm{~nm}) .{ }^{89,90}$ Moreover, the TA features decay in all complexes with lifetimes of a few picoseconds (2.5-4 ps) and a few tens of picoseconds (22-28 ps, Tables S8-S9), with the dynamics of the ESA and GSB bands mirroring each other, meaning that we are probing the same exciton population (Figures $6 \mathrm{~b}$ and S31 and S32). The fast exciton quenching (vs a lifetime of hundreds of picoseconds in CPT alone) could be due to additional nonradiative deactivation paths offered by the specific interactions with the ssDNA and energy dissipation to the environment, ${ }^{91,92}$ or to charge transfer followed by ultrafast recombination (since no TA signatures of polarons are seen). ${ }^{55,89}$ Finally, no long-lived species are formed in the complexes contrary to CPT alone in solution, where long-lived polarons are generated at low temperature and intersystem crossing populates the triplet state at high temperature. ${ }^{55}$ Along with the fast exciton quenching, this is additional proof that the assembly of CPT with the different ssDNA sequences is always achieved and leads to isolated CPT chains, as polaron formation is usually observed in polythiophenes with interchain interactions. ${ }^{55}$ The absence of triplet generation provides additional support for the higher torsional order of $\mathrm{CPT}$ in all complexes, as intersystem crossing is aided by torsional disorder.

With the knowledge that only one excited state species is present (intrachain delocalized excitons), we can use RRIA to evaluate the early time structural evolution of CPT in the various complexes. RRIA requires first the quantification of 
resonance Raman cross sections $\left(\sigma_{\mathrm{R}}\right.$; section S9.1.1). The absolute $\sigma_{\mathrm{R}}$ 's for all the RR bands of CPT were calculated for excitation at $473 \mathrm{~nm}$ for $\mathrm{CPT} / \mathrm{T} 406$ and $\mathrm{CPT} / \mathrm{dA}_{20}$ and at 532 $\mathrm{nm}$ for CPT/ $\mathrm{dC}_{20}$ (Table S10), which is always $\sim 1900 \mathrm{~cm}^{-1}$ to the blue side of the $\lambda_{\max }$ of the absorption spectrum. These cross sections along with the absorption cross section for each complex were then simultaneously modeled (see sections S9.1.2-3). The fits to the absorption spectra are shown in Figure 2, while Figures S33-S35 show the RR excitation profiles (REP's) for CPT in all the complexes, i.e., the calculated $\sigma_{\mathrm{R}}$ as a function of excitation energy. The fitting parameters are reported in Table S11. We find that the $\mathrm{C}_{\beta^{\prime}}-\mathrm{C}_{\beta}$ stretch exemplifies the highest displacement $(\Delta)$ in $\mathrm{CPT} / \mathrm{dC}_{20}$ compared to other modes, i.e., the largest change in bond length upon excitation, while the $\mathrm{C}_{\alpha}=\mathrm{C}_{\beta}$ symmetric stretch (also reflecting the transition toward a quinoidal state) becomes increasingly important as we move from $\mathrm{CPT} / \mathrm{dC}_{20}$ to $\mathrm{CPT} / \mathrm{dA}_{20}$, with $\Delta$ about 3 times as large in the latter complex (Figure 7a). The total reorganization energy, $\lambda$, calculated from mode-specific reorganization energies $\left(\lambda_{\text {tot }}=\right.$ $\left.\left.\sum \lambda_{i}=\sum_{i} \omega_{i} \Delta_{i} / 2\right)\right)$, also demonstrates a 3 times larger structural evolution on going from the ground to the excited state in the case of $\mathrm{dA}_{20}\left(\lambda=1119 \mathrm{~cm}^{-1}\right.$ for $\mathrm{dC}_{20}, 1627 \mathrm{~cm}^{-1}$ for $\mathrm{T} 406$, and $3024 \mathrm{~cm}^{-1}$ for $\mathrm{dA}_{20}$, see Figure $\left.7 \mathrm{a}\right)$. In addition, we see doubling of the inhomogeneous broadening from 450 $\mathrm{cm}^{-1}(56 \mathrm{meV})$ in $\mathrm{CPT} / \mathrm{dC}_{20}$ to $900 \mathrm{~cm}^{-1}(112 \mathrm{meV})$ in $\mathrm{CPT} / \mathrm{dA}_{20}$, indicating the larger number of energetic sites available due to conformational disorder. This illustrates an overall larger conformational rearrangement in response to the change of electron density in the excited state for the more flexible complexes, where our RR measurements and the MD simulations show that the polymer is less tightly bound to the ssDNA and the CPT conformation is less planar in the ground state with more torsional disorder.

Structural changes in the excited state are also reflected by the temporal evolution of the spectral shapes from TA spectroscopy. In particular, the clear vibronic structure of the $\mathrm{TA}$ spectra of $\mathrm{CPT} / \mathrm{dC}_{20}$ in the $\mathrm{GSB} / \mathrm{SE}$ region allows isolation of the time-dependent $\mathrm{SE}$ spectra after subtracting the properly scaled calculated absorption (Figure $7 \mathrm{~b}$ ) and use of a similar methodology to RRIA to analyze them (Figure $7 \mathrm{c}$, section S9.1.3). The earliest SE spectrum accessible within the time resolution of our TA experiment (at $200 \mathrm{fs}$ ) already displays a Stokes shift of $52 \mathrm{meV}(\sim 70 \%$ of the total Stokes shift) with respect to the absorption. Such behavior in conjugated polymers is typically related to ultrafast dynamic localization of the initial photoexcitation, caused by electronic relaxation and coupling to nuclear modes. ${ }^{56,93-97}$ However, the initial Stokes shift observed for $\mathrm{CPT} / \mathrm{dC}_{20}$ is less pronounced than in other systems and (in contrast to, e.g., P3HT) ${ }^{56}$ the early emission spectrum is a mirror image of the absorption that can be reproduced with identical parameters. This points to limited (mainly electronic) relaxation within $<200 \mathrm{fs}$, maintaining delocalization of the excited state and possibly favoring the occurrence of coherent exciton migration along the chain. $15,95,97,98$

At longer time delays ( $>200 \mathrm{fs}$ ), the SE spectrum of CPT/ $\mathrm{dC}_{20}$ undergoes a red-shift of the $0-0$ band (by $\sim 22 \mathrm{meV}$, leading to a total Stokes shift of $74 \mathrm{meV}$ ), and an increase of the vibronic band intensitiy ratio $\left(I_{0-0} / I_{0-1}\right)$ from 1.34 at 0.2 ps to 3.41 at 10 ps (Figure $7 \mathrm{~b}$ ). Fitting of the SE spectra reveals that the geometrical relaxation of the polymer involves mainly the $\mathrm{C}_{\beta}-\mathrm{C}_{\beta^{\prime}}$ stretch, as this exhibits the highest contribution to the SE vibronic progression and sustains the largest reduction in $\Delta$ with time (Figure $7 \mathrm{~d}$, Table S12). A concomitant decrease of the inhomogeneous broadening, which indicates a narrower distribution of chromophores, occurs within the first 2 ps. On the basis of previous literature about polythiophenes, this slower relaxation is ascribed to mainly incoherent exciton migration to lower energy chain segments (longer, more planar), accompanied by torsional relaxation of the backbone. ${ }^{13,56,99-101}$ Again, the effect in $\mathrm{CPT} / \mathrm{dC}_{20}$ is weaker and faster than generally observed (e.g., the total Stokes shift for polythiophene solutions is usually twice as large and spectral relaxation proceeds up to $100-200 \mathrm{ps}) .{ }^{56,99}$ The limited geometrical relaxation in the complex agrees with the small reorganization energy calculated by RRIA $(\sim 140 \mathrm{meV})$ and the fact that $\mathrm{dC}_{20}$ induces a more planar and tighter conformation of CPT already in the ground state, hindering large reorganization of the excited state. Moreover, exciton migration is limited to intrachain processes and likely concerns a more homogeneous distribution of chain segments with similar energy. Going to $\mathrm{CPT} / \mathrm{dA}_{20}$, a slightly more important spectral red-shift is observed in the negative TA features (from $593 \mathrm{~nm}$ at 0.2 ps to $600 \mathrm{~nm}$ at $5 \mathrm{ps}$, vs from 591 to $594 \mathrm{~nm}$ in $\left.\mathrm{CPT} / \mathrm{dC}_{20}\right)$, as predicted by the higher reorganization energy in the more flexible complex. This is still much less than the red-shift of the TA bands observed for random-coiled CPT alone at $55{ }^{\circ} \mathrm{C}(40 \mathrm{~nm}$ shift over $100 \mathrm{ps}),{ }^{55}$ suggesting that even for $\mathrm{CPT} / \mathrm{dA}_{20}$ the excited-state relaxation is restricted by the complexation with ssDNA.

\section{CONCLUSIONS}

We have investigated here the templating effect of ssDNA oligomers with different sequences on cationic polythiophenes. The conformational and photophysical behavior of the polymer/ssDNA complexes was studied using a powerful combination of spectroscopic techniques supported by $\mathrm{MD}$ simulations, leading to significant expansion of knowledge and understanding of the systems. We find that in all complexes, strong electrostatic interactions develop between the two components, leading to reduced polymer aggregation so that the properties of isolated chains can be accessed. However, large variations in the templating effect between different polymer/ssDNA combinations show the importance of additional noncovalent interactions. We identify $\mathrm{CPT} / \mathrm{dC}$ as the complex displaying the most pronounced templating effect, highlighted by the most extensive intrachain coupling and optical response of the polymer. The first key to this effective templating is the extensive succession of cytosines in $\mathrm{dC}$ (ideally $10-20$ bases), which favors $\pi$-stacking with the thiophene/imidazole rings of CPT over strong intra-DNA interactions, inducing a more planar and tighter conformation of the polymer. In contrast, CPT interacts mainly electrostatically with $\mathrm{dA}_{20}$ (H-bonding and $\pi$-stacking are weak), allowing conformational flexibility of the polymer, while $\mathrm{dA}_{20}$ maintains a rigid helical structure in the complex due to strong adenineadenine stacking. Mixed ssDNA sequences induce a variable degree of order in $\mathrm{CPT}$, depending on the intricate interplay of ssDNA-CPT and intra-DNA interactions, but never approaching the one of $\mathrm{dC}_{20}$. We find that the second key to effective templating resides in the nature of the polymer side chains. Substituting CPT with another imidazole-based polythiophene (P3HT-Im) causes poor templating even with $\mathrm{dC}_{20}$, because the cationic side chains are in this case too long to induce a good conformational fit with the ssDNA, and the absence of an 
additional methyl group on the thiophenes enhances torsional disorder on the backbone.

Finally, we show that supramolecular assembly with ssDNA strongly affects the excited-state properties of CPT, leading to intrachain delocalized excitons with a relatively short lifetime and the absence of any long-lived polaron or triplet states. In addition, excited-state structural relaxation is significantly reduced, with the rigid $\mathrm{CPT} / \mathrm{dC}_{20}$ complex having a particularly small reorganization energy. This maintains intrachain exciton delocalization, favoring (possibly coherent) directional exciton migration along the chains. Linearity of the conjugated chain previously allowed isolated polydiacetylene (PDA) to function as electrical conductive nanowires due to the ability to transmit charge over long distances by coherent tunnelling, ${ }^{102,103}$ or to exhibit efficient and unidirectional coherent excitonic energy transfer due to delocalized states over the chain. ${ }^{104}$ In a comparable approach, the templating effect of ssDNA can provide access to versatile applications in optoelectronics ranging from artificial light-harvesting to single-molecule circuits based on careful design of supramolecular assemblies with conjugated polymers.

\section{EXPERIMENTAL SECTION}

Materials. CPT (Figure 1) was synthesized as previously described. $^{51,105}$ It has a molecular weight $(\mathrm{Mw})$ of $22 \mathrm{kDa}(\mathrm{Mn}=$ $11 \mathrm{kDa}$ ), with a polydispersity index (PDI) of 2.0. Each monomer unit of $262.8 \mathrm{~g} / \mathrm{mol}$ contains one positive charge on the ionic sidechain, compensated by a $\mathrm{Cl}^{-}$counterion. Cationic poly [3-(6'(trimethylphosphonium)hexyl)thiophene-2,5-diyl] (P3HT-PMe ${ }_{3}$ ) and poly[3-(6'-(imidazolium)hexyl)thiophene-2,5-diyl] (P3HT-Im; Figure $5 \mathrm{~d}$ ) were synthesized as previously described. ${ }^{58} \mathrm{P}^{\mathrm{H}} \mathrm{HT}-\mathrm{PMe}_{3}$ and P3HT-Im have number-averaged molecular weight $(\mathrm{Mn})$ of 17.7 $\mathrm{kDa}$ and $18.1 \mathrm{kDa}(\mathrm{PDI}=1.27)$, and the molecular weight of each monomer unit is $320.01 \mathrm{~g} / \mathrm{mol}$ and $326.117 \mathrm{~g} / \mathrm{mol}$, respectively. Each monomer unit is compensated by a $\mathrm{Br}^{-}$counterion. A stock solution of each polymer (monomeric concentration of $2 \times 10^{-3} \mathrm{M}$ ) was prepared in water (purified through a Synergy water purification system) and stored in the freezer. Single-stranded oligonucleotides (ssDNAs) were purchased from Sigma-Aldrich. The ssDNAs with a length of 20 monomers are named $\mathrm{dA}_{20}, \mathrm{dG}_{20}$ (homopurine), T406, T406RC, d $\left[(\text { GGAA })_{5}\right], \mathrm{d}\left[\left(\mathrm{TTCC}_{5}\right], \mathrm{d}\left[\left(\mathrm{AT}(\mathrm{TAT})_{6}\right], \mathrm{d}\left[\mathrm{AT}(\mathrm{AAT})_{6}\right]\right.\right.$ (mixed sequence), $\mathrm{dT}_{20}$, and $\mathrm{dC}_{20}$ (homopyrimidine). All oligonucleotides were previously used by Charlebois et al., ${ }^{52}$ and their sequences are listed in the Table S1. A stock solution of each ssDNA (monomeric concentration of $2 \times 10^{-3} \mathrm{M}$ ) was prepared in water and stored in the freezer. Phosphate buffered saline (PBS) was purchased from Thermo Fisher Scientific (catalogue number: 10010-031, $\mathrm{pH}$ 7.4, $\mathrm{KH}_{2} \mathrm{PO}_{4} 1.06 \mathrm{mM}, \mathrm{Na}_{2} \mathrm{HPO}_{4} 2.97 \mathrm{mM}, \mathrm{NaCl} 155 \mathrm{mM}$ ) and was used to dilute polymer and ssDNA stock solutions to different monomeric concentrations depending on the experiment: $7.3 \times 10^{-5}$ $\mathrm{M}$ for stationary absorption and $\mathrm{CD}$ measurements, $1.5 \times 10^{-4} \mathrm{M}$ for TA and RR experiments in $\mathrm{dA}_{20}$ and T406, and $3 \times 10^{-4} \mathrm{M}$ for RR experiments in $\mathrm{dC}_{20}$. For cationic polythiophene/ssDNA experiments, solutions with monomeric equivalence were prepared, with one positive charge from cationic polythiophene for one negative charge of ssDNA in order to have a complete formation of the complex form (see titration in Figure S19). ${ }^{51,52}$ The order of addition for all the solutions was the following: PBS (solvent), cationic polythiophene, ssDNA. We note that the $\mathrm{CPT} / \mathrm{dC}_{20}$ complex was formed and studied at $55^{\circ} \mathrm{C}$ only for the stationary absorption and $\mathrm{CD}$ measurements. In order to avoid precipitation effects during the longer RR and TA measurements, the $\mathrm{CPT} / \mathrm{dC}_{20}$ complex was formed and studied at 20 ${ }^{\circ} \mathrm{C}$, although this decreases the $A_{0-0} / A_{0-1}$ ratio to 1.16 (see Figure $\mathrm{S} 3 \mathrm{~b})$.

For experiments with different lengths of $\mathrm{dC}$, aqueous solutions (0.1 mM, oligomer concentration) of homocytosine of five different lengths $(5,10,20,40$, and 80 bases $)$ were also purchased from Sigma-
Aldrich. The homocytosine oligomers and their monomeric concentrations are listed in Table S2. PBS was used to dilute the CPT and ssDNA stock solutions. CPT first: $58.4 \mu \mathrm{L}$ of CPT $(2 \times$ $10^{-3} \mathrm{M}$ ) was added to $1540 \mu \mathrm{L}$ of PBS to obtain a final concentration of $7.3 \times 10^{-5} \mathrm{M}$, on a monomeric basis. Then, several portions of $\mathrm{dC}$ (each portion corresponding to $1 / 4$ of the total monomeric concentration of $\mathrm{CPT}$ ) were added to the $\mathrm{CPT}$ solution. $\mathrm{dC}$ first: a volume of $\mathrm{dC}$ stock solution (different for each $\mathrm{dC}$ ) was added to $1540 \mu \mathrm{L}$ of PBS to obtain a final concentration of $7.3 \times 10^{-5} \mathrm{M}$, on a monomeric basis. Then several portions of $14.6 \mu \mathrm{L}$ of CPT $\left(2 \times 10^{-3}\right.$ $\mathrm{M}$, corresponding to $1 / 4$ of the total monomeric concentration of $\mathrm{dC})$ were added to the $\mathrm{dC}$ solution. After each addition of every titration, the solution was left to equilibrate for at least 5-10 min before recording the steady-state absorption and CD spectra. The temperature was set at $20^{\circ} \mathrm{C}$.

For the study of the $\mathrm{pH}$ dependence of the stability of i-motif conformation for $\mathrm{dC} 20$, minor portions of $0.1 \mathrm{M} \mathrm{HCl}$ or $\mathrm{NaOH}$ were added, either to lower or raise $\mathrm{pH}$, respectively. The $\mathrm{pH}$ titrations were carried out at $20^{\circ} \mathrm{C}$ using $\mathrm{H}_{2} \mathrm{O}, \mathrm{NaCl}$, or PBS buffer as a solvent for salt-dependence studies. The reported $\mathrm{pH}$ values (measured with accuracy of $\pm 0.05 \mathrm{pH}$ units) are those obtained before the $\mathrm{CD}$ spectra were taken.

Absorption Spectroscopy. Absorption spectra were recorded with a UV/vis/NIR Lambda 900 spectrometer (PerkinElmer). A quartz cuvette with an optical path length of $10 \mathrm{~mm}$ was placed inside the stand-alone Peltier-based temperature-controlled cuvette holder (Flash300/E, Quantum Northwest), and the temperature was allowed to stabilize for 5-10 min. A small magnetic stir bar was placed in the cuvette, and the stirring speed could be controlled with the cuvette holder.

Circular Dichroism Spectroscopy. CD spectra were recorded with a J-715 spectropolarimeter (Jasco). A quartz cuvette with an optical path length of $10 \mathrm{~mm}$ was placed inside the temperaturecontrolled holder of the spectropolarimeter. For the $\mathrm{pH}$ titration of $\mathrm{dC}_{20}$, CD spectra were recorded with a Jasco J-815 CD Spectrometer using EPR Suprasil tubes (diameter: $4 \mathrm{~mm}$ ).

Transient Absorption Spectroscopy. TA spectra were recorded using femtosecond pulsed laser pump-probe spectroscopy. The solutions were placed in a quartz cuvette with an optical path length of $2 \mathrm{~mm}$ (Starna Cells Inc.) placed inside the temperature-controlled holder and held by a piece of aluminum. Pump excitation at $400 \mathrm{~nm}$ (200 fs resolution) was achieved by frequency doubling the fundamental $800 \mathrm{~nm}$ laser output (from a Ti:sapphire laser system with regenerative amplification providing $35 \mathrm{fs}$ pulses at a repetition rate of $1 \mathrm{kHz}$, Astrella, Coherent). As an alternative, an excitation beam at $580 \mathrm{~nm} \quad(<100$ fs resolution) was generated with a commercial optical parametric amplifier (OPerA Solo, Coherent). The pump diameter was about $1 \mathrm{~mm}$, and the pump intensity was 400 $\mathrm{nJ}$ with the $400 \mathrm{~nm}$ excitation and between 200 and $330 \mathrm{~nJ}$ for the $580 \mathrm{~nm}$ excitation. The probe beam consisted of a white light continuum $(\sim 450-1200 \mathrm{~nm})$ generated by passing a portion of the $800 \mathrm{~nm}$ amplified Ti:sapphire output through a $5 \mathrm{~mm}$ thick sapphire window. Either $720 \mathrm{~nm}$ low pass filters or $850 \mathrm{~nm}$ long pass filters were used to remove the remaining fundamental intensity from the white light, when the visible and the near-infrared (nIR) parts of the spectrum were recorded separately. The probe intensity was negligible compared to the pump intensity, and the spot size was much smaller (probe energy of $<5 \mathrm{~nJ}$, probe diameter of about $160 \mu \mathrm{m}$ ). The probe pulses were time-delayed with respect to the pump pulses by means of a computer-controlled translation stage. The probe beam was split before the sample into a signal beam (transmitted through the sample and overlapped with the pump beam) and a reference beam. The signal and reference beams were detected separately using a pair of spectrographs (home-built prism spectrometers) equipped with $512 \times$ 58 pixel back-thinned CCDs (Hamamatsu S07030-0906) and assembled by Entwicklungsbüro Stresing, Berlin. The pump beam was chopped at half the amplifier frequency to improve the sensitivity of the setup. The transmitted intensity of the signal beam was recorded shot-by-shot, and it was corrected for laser intensity fluctuations using the reference beam. The single shot TA spectra 
were averaged 3000 times at each time delay, and the entire range of measured time delays (between -4 ps and $1 \mathrm{~ns}$ ) was scanned five times, without any noticeable signs of degradation. Wavelength calibration was accomplished with a series of $10 \mathrm{~nm}$ bandpass filters. To avoid polarization effects, the relative polarization of the probe and pump pulses was set at the magic angle. All spectra were corrected for the chirp of the white-light probe. MATLAB and IgorPro software were used for data analysis.

Resonance Raman Studies. Resonance Raman (RR) spectra of cationic polythiophene/ssDNA complexes were obtained with excitation at 435.69, 473, 532, and $266 \mathrm{~nm}$. The 532 and $266 \mathrm{~nm}$ excitation wavelengths employed in the $R R$ experiments were provided by the second and fourth harmonics of a Q-switched Nd:YAG laser (PRO-230, $30 \mathrm{~Hz}$, Spectra Physics), and the $435.69 \mathrm{~nm}$ was produced via Raman shifting at $532 \mathrm{~nm}$ in a $1 \mathrm{~m}$ tube containing $\mathrm{H}_{2}$ gas. The $473 \mathrm{~nm}$ excitation was obtained from a CW diode laser (Ultralasers, $50 \mathrm{~mW}$ OEM DPSS Laser). The excitation light was focused into a spinning cell consisting of an EPR Suprasil tube (diameter: $4 \mathrm{~mm}$ ) attached to a rheostat-controlled motor for choice of rotation speed. Use of the spinning cell prolonged the lifetime of the samples. Modest excitation energies $(2.1 \mathrm{~mW}$ at 473 and 435.69 $\mathrm{nm}, 0.1 \mathrm{~mW}(3.3 \mu \mathrm{J}$ per pulse $)$ at $532 \mathrm{~nm}$, and $0.07 \mathrm{~mW}$ at $266 \mathrm{~nm}$ $(\sim 2.5 \mu \mathrm{J}$ per pulse)) were employed to avoid decomposition of the sample, which was monitored by obtaining the absorption spectrum of the sample before and after exposure. The Raman scattered light was collected in a backscattering geometry and delivered to a $0.75 \mathrm{~m}$ focal-length Czerny-Turner spectrograph, equipped with a 1200grooves/mm holographic grating for the visible wavelengths and a 2400 grooves $/ \mathrm{mm}$ holographic grating for excitation at $266 \mathrm{~nm}$. The slit width was set to $100 \mu \mathrm{m}$ providing for $5 \mathrm{~cm}^{-1}$ spectral resolution at the visible wavelengths used in this work and $7 \mathrm{~cm}^{-1}$ at $266 \mathrm{~nm}$. The scattered light was detected by a $\mathrm{LN}_{2}$-cooled $2048 \times 512$ pixel, back-illuminated UV-enhanced CCD detector (Spec10:2KBUV/LN, Princeton Instruments). Each spectrum with excitation in the visible is the accumulation of $6 \times 5$ min spectra, while $20 \times 10 \mathrm{~min}$ spectra were required in the UV. Frequency calibration of the spectra was accomplished with the use of cyclohexane. MATLAB and ORIGIN software were used for spectral treatment and analysis.

\section{COMPUTATIONAL METHODS}

MD Simulations. For the ssDNA fragments only, the system featured the respective ssDNA 20 mer $\left(\mathrm{dC}_{20}\right.$ or $\left.\mathrm{dA}_{20}\right)$, approximately 5000 water molecules and $20 \mathrm{Na}^{+}$counterions for charge neutralization. The parmbsc1 force field ${ }^{106,107}$ was used for ssDNA, while water was modeled with the TIP $3 \mathrm{P}^{108}$ force field with corresponding parameters for $\mathrm{Na}^{+}$ions. ${ }^{109}$ Both systems were first equilibrated at $300 \mathrm{~K}$ and $1 \mathrm{~atm}$, and then the production phase was carried out for a total of $1.8 \mu \mathrm{s}$, with a time step of $2 \mathrm{fs}$. Bonds involving $\mathrm{H}$ atoms were kept fixed using the SHAKE algorithm.

For all CPT/ssDNA assemblies, two separate simulations were performed for each system with different initial distances between the ssDNA and the polymer (10 and $30 \AA$, respectively). The resulting periodic boxes contained approximately 30000 and 54000 water molecules, respectively. In addition to the solvent and the ssDNA and CPT 20mers, the system setup for both simulations also included a biologically relevant $150 \mathrm{mM}$ concentration of $\mathrm{Na}^{+}$and $\mathrm{Cl}^{-}$ions, in line with the ionic strength of the PBS buffer solution employed in the experiments. The ssDNA 20mers, water, and ions were described in the same fashion as in the pure ssDNA simulations. Point charges for the polymer atoms (listed in detail in Figure S1 and Tables S3 and S4) were obtained from static calculations performed on CPT tetramers, using Gaussian 09, ${ }^{110}$ and the polarizable continuum model for water. ${ }^{111,112}$ The geometry of the tetramers was optimized at the Density Functional Theory (DFT) level, ${ }^{113,114}$ employing the B3LYP functional ${ }^{115,116}$ combined with the $6-31 \mathrm{G}(\mathrm{d}, \mathrm{p})$ basis set, ${ }^{117,118}$ recently used for a study of another polythiophene derivative. ${ }^{119}$ Atom types and interactions between CPT atoms were assigned according to the recently updated General AMBER force field $(\text { GAFF2 })^{120,121}$ with the exception of the S-C-C-S torsion angle where parameters were selected from ref 119 . The simulation protocol was the same as for the pure ssDNA simulations, with the production phase amounting to a total of $1.54 \mu \mathrm{s}$ from the two independent MD runs. All simulations were carried out with the GPU version of AMBER 16. ${ }^{122}$

A simulation for CPT in aqueous solution (with approximately 25000 water molecules and $150 \mathrm{mM} \mathrm{NaCl}$ ) was also carried out. The aforementioned parametrization for $\mathrm{CPT}, \mathrm{H}_{2} \mathrm{O}, \mathrm{Na}^{+}$, and $\mathrm{Cl}^{-}$was used. The simulation protocol used for the DNA and CPT/DNA systems was also employed here, amounting to $1.5 \mu \mathrm{s}$ of total simulation time in the production phase.

Chirality Analysis. For two neighboring nucleobases, $i$ and $i+1$, their local chirality index, $\mathrm{CI}_{i, i+1}$, is defined as

$$
\mathrm{CI}_{i, i+1}=\frac{\vec{r}_{i, i+1} \cdot\left(\vec{\mu}_{i} \times \vec{\mu}_{i+1}\right)}{\left|\vec{r}_{i, i+1}\right| \cdot\left|\vec{\mu}_{i}\right| \cdot\left|\vec{\mu}_{i+1}\right|}
$$

In eq $1, \vec{r}_{i, i+1}$ is the vector connecting the centers of mass of the nucleobases $i$ and $i+1$, while $\vec{\mu}_{i}$ and $\vec{\mu}_{i+1}$ are unit vectors that are perpendicular to their respective planes. The average local chirality index for a given frame of the trajectory is then obtained by averaging the indices over the entire length of the ssDNA.

\section{ASSOCIATED CONTENT}

\section{Supporting Information}

The Supporting Information is available free of charge at https://pubs.acs.org/doi/10.1021/acs.chemmater.0c02251.

Additional computational and spectral data (absorption, resonance Raman, CD, transient absorption) and resonance Raman intensity analysis. Data shown in the main figures are available at https://boris.unibe.ch/id/ eprint/146048 (PDF)

\section{AUTHOR INFORMATION}

\section{Corresponding Authors}

Sophia C. Hayes - Department of Chemistry, University of Cyprus, 1678 Nicosia, Cyprus; 1 orcid.org/0000-0003-02386915; Email: shayes@ucy.ac.cy

Natalie Banerji - Department of Chemistry and Biochemistry,

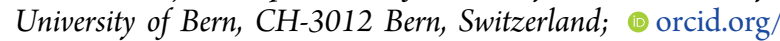
0000-0001-9181-2642; Email: natalie.banerii@dcb.unibe.ch

Ursula Rothlisberger - Laboratory of Computational Chemistry and Biochemistry, Ecole Polytechnique Féderale de Lausanne (EPFL), CH-1015 Lausanne, Switzerland; (1) orcid.org/0000-0002-1704-8591; Email: ursula.roethlisberger@epfl.ch

\section{Authors}

Lisa Peterhans - Department of Chemistry and Biochemistry, University of Bern, CH-3012 Bern, Switzerland

Eliana Nicolaidou - Department of Chemistry, University of Cyprus, 1678 Nicosia, Cyprus

Polydefkis Diamantis - Laboratory of Computational Chemistry and Biochemistry, Ecole Polytechnique Féderale de Lausanne (EPFL), CH-1015 Lausanne, Switzerland; - o orcid.org/0000-0003-0535-3158

Elisa Alloa - Department of Chemistry, University of Cyprus, 1678 Nicosia, Cyprus

Mario Leclerc - Department of Chemistry, Universite Laval, G1K 7P4 Quebec City, Quebec, Canada; 으잉.org/00000003-2458-9633

Mathieu Surin - Laboratory for Chemistry of Novel Materials, Center for Innovation in Materials and Polymers, University of Mons - UMONS, B-7000 Mons, Belgium; 이이이.org/00000001-8950-3437 
Sébastien Clément - Institut Charles Gerhardt Montpellier, ICGM, UMR 5253 CNRS, Université de Montpellier, F-34095 Montpellier, Cedex 05, France; ๑ orcid.org/0000-0002-84738197

Complete contact information is available at: https://pubs.acs.org/10.1021/acs.chemmater.0c02251

\section{Author Contributions}

${ }^{\perp}$ L.P. and E.N. equally contributed to this work. CPT polymer was synthesized by M.L.'s group and P3HT-PMe 3 and P3HTIm polymers were synthesized and provided by S.C.'s and M.S.'s groups. Spectroscopic data were collected by L.P., E.N. and E.A. and analyzed by L.P., E.N., S.C.H. and E.A. The experiments were conceived and supervised by N.B and S.C.H. P.D. conducted and analyzed the MD simulations which were supervised by U.R. All the authors discussed the results. N.B. and S.C.H. wrote the manuscript with input from all other authors.

\section{Notes}

The authors declare no competing financial interest.

\section{ACKNOWLEDGMENTS}

S.C.H. and N.B. thank Prof. Carlos Silva (Georgia Tech) and Prof. Epameinondas Leontidis (University of Cyprus) for useful discussions. S.C.H. and E.N. would like to thank the University of Cyprus for financial support. L.P. and N.B. thank the European Research Council (ERC) for supporting this research by a Starting Grant (No. 714586, OSIRIS), as well as the University of Bern. S.C.H and E.A. acknowledge financial support from the EU Horizon 2020 Research and Innovation Programme, SYNCHRONICS Project (Grand Agreement 643238). U.R and N.B acknowledge NCCR-MUST, a research instrument of the Swiss National Science Foundation. Research in Mons was supported by the Fund for Scientific Research (F.R.S.-FNRS) under grants MIS F.4532.16 and EOS 30650939. Research in Montpellier is supported by CNRS and Université de Montpellier.

\section{REFERENCES}

(1) Guo, X.; Baumgarten, M.; Müllen, K. Designing $\pi$-Conjugated Polymers for Orgnnnnanic Electronics. Prog. Polym. Sci. 2013, 38 (12), 1832-1908.

(2) Jeng, K. S.; Chu, C. W.; Liu, C. L.; Jean, W. M.; Chen, H. L.; Chen, J. T. Orientation Preferences of Interchain Stackings for Poly(3-Hexylthiophene) Nanowires Prepared Using Template-Based Wetting Methods. Macromol. Chem. Phys. 2018, 219 (11), 1800078.

(3) Van Den Eede, M. P.; Van Gestel, L.; Koeckelberghs, G. Expression of Chirality in Tailor-Made Conjugated Polymers. Macromolecules 2018, 51 (17), 6602-6608.

(4) Zhu, C.; Fang, L. Locking the Coplanar Conformation of $\pi$ Conjugated Molecules and Macromolecules Using Dynamic Noncovalent Bonds. Macromol. Rapid Commun. 2018, 39 (2), 1700241.

(5) Zhu, C.; Mu, A. U.; Lin, Y. H.; Guo, Z. H.; Yuan, T.; Wheeler, S. E.; Fang, L. Molecular Coplanarity and Self-Assembly Promoted by Intramolecular Hydrogen Bonds. Org. Lett. 2016, 18 (24), 63326335.

(6) Raithel, D.; Simine, L.; Pickel, S.; Schötz, K.; Panzer, F.; Baderschneider, S.; Schiefer, D.; Lohwasser, R.; Köhler, J.; Thelakkat, M.; et al. Direct Observation of Backbone Planarization via SideChain Alignment in Single Bulky-Substituted Polythiophenes. Proc. Natl. Acad. Sci. U. S. A. 2018, 115 (11), 2699-2704.

(7) Dyson, M. J.; Lariou, E.; Martin, J.; Li, R.; Erothu, H.; Wantz, G.; Topham, P. D.; Dautel, O. J.; Hayes, S. C.; Stavrinou, P. N.; et al.
Managing Local Order in Conjugated Polymer Blends via Polarity Contrast. Chem. Mater. 2019, 31 (17), 6540-6547.

(8) Sugimoto, T.; Ebihara, Y.; Ogino, K.; Vacha, M. StructureDependent Photophysics Studied in Single Molecules of Polythiophene Derivatives. ChemPhysChem 2007, 8 (11), 1623-1628.

(9) Hollingsworth, W. R.; Segura, C.; Balderrama, J.; Lopez, N.; Schleissner, P.; Ayzner, A. L. Exciton Transfer and Emergent Excitonic States in Oppositely-Charged Conjugated Polyelectrolyte Complexes. J. Phys. Chem. B 2016, 120 (31), 7767-7774.

(10) Kanemoto, K.; Sudo, T.; Akai, I.; Hashimoto, H.; Karasawa, T.; Aso, Y.; Otsubo, T. Intrachain Photoluminescence Properties of Conjugated Polymers as Revealed by Long Oligothiophenes and Polythiophenes Diluted in an Inactive Solid Matrix. Phys. Rev. B: Condens. Matter Mater. Phys. 2006, 73 (23), 235203.

(11) Adachi, T.; Lakhwani, G.; Traub, M. C.; Ono, R. J.; Bielawski, C. W.; Barbara, P. F.; Vanden Bout, D. A. Conformational Effect on Energy Transfer in Single Polythiophene Chains. J. Phys. Chem. B 2012, 116 (32), 9866-9872.

(12) Song, Y.; Hellmann, C.; Stingelin, N.; Scholes, G. D. The Separation of Vibrational Coherence from Ground- and ExcitedElectronic States in P3HT Film. J. Chem. Phys. 2015, 142 (21), 212410.

(13) Park, K. H.; Kim, W.; Yang, J.; Kim, D. Excited-State Structural Relaxation and Exciton Delocalization Dynamics in Linear and Cyclic $\pi$-Conjugated Oligothiophenes. Chem. Soc. Rev. 2018, 47 (12), 42794294.

(14) Portone, A.; Ganzer, L.; Branchi, F.; Ramos, R.; Caldas, M. J.; Pisignano, D.; Molinari, E.; Cerullo, G.; Persano, L.; Prezzi, D.; Virgili, T. Tailoring Optical Properties and Stimulated Emission in Nanostructured Polythiophene. Sci. Rep. 2019, 9 (1), 1-10.

(15) Binder, R.; Lauvergnat, D.; Burghardt, I. Conformational Dynamics Guides Coherent Exciton Migration in Conjugated Polymer Materials: First-Principles Quantum Dynamical Study. Phys. Rev. Lett. 2018, 120 (22), 2-5.

(16) Hamedi, M.; Elfwing, A.; Gabrielsson, R.; Inganäs, O. Electronic Polymers and DNA Self-Assembled in Nanowire Transistors. Small 2013, 9 (3), 363-368.

(17) Sun, C.; Mróz, M. M.; Castro Smirnov, J. R.; Lüer, L.; Hermida-Merino, D.; Zhao, C.; Takeuchi, M.; Sugiyasu, K.; Cabanillas-González, J. Amplified Spontaneous Emission in Insulated Polythiophenes. J. Mater. Chem. C 2018, 6 (24), 6591-6596.

(18) Grozema, F. C.; Siebbeles, L. D. A. Introduction: Molecular Electronics and Molecular Wires. In Charge and Exciton Transport through Molecular Wires; John Wiley \& Sons, Ltd, 2011; pp 1-15.

(19) Li, C.; Bai, H.; Shi, G. Conducting Polymer Nanomaterials: Electrosynthesis and Applications. Chem. Soc. Rev. 2009, 38 (8), 2397-2409.

(20) Liu, R.; Lee, S. B. MnO2/Poly(3,4-Ethylenedioxythiophene) Coaxial Nanowires by One-Step Coelectrodeposition for Electrochemical Energy Storage. J. Am. Chem. Soc. 2008, 130 (10), 29422943.

(21) Ambade, R. B.; Ambade, S. B.; Shrestha, N. K.; Salunkhe, R. R.; Lee, W.; Bagde, S. S.; Kim, J. H.; Stadler, F. J.; Yamauchi, Y.; Lee, S. H. Controlled Growth of Polythiophene Nanofibers in $\mathrm{TiO}_{2}$ Nanotube Arrays for Supercapacitor Applications. J. Mater. Chem. A 2017, 5 (1), 172-180.

(22) Andrew, T. L.; Swager, T. M. Structure Property Relationships for Exciton Transfer in Conjugated Polymers. In Charge and Exciton Transport through Molecular Wires; John Wiley \& Sons, Ltd: Weinheim, Germany, 2011; Chapter 10, pp 271-310.

(23) Boulais, E.; Sawaya, N. P. D.; Veneziano, R.; Andreoni, A.; Banal, J. L.; Kondo, T.; Mandal, S.; Lin, S.; Schlau-Cohen, G. S.; Woodbury, N. W.; et al. Programmed Coherent Coupling in a Synthetic DNA-Based Excitonic Circuit. Nat. Mater. 2018, 17, 159166.

(24) Haedler, A. T.; Kreger, K.; Issac, A.; Wittmann, B.; Kivala, M.; Hammer, N.; Köhler, J.; Schmidt, H. W.; Hildner, R. Long-Range Energy Transport in Single Supramolecular Nanofibres at Room Temperature. Nature 2015, 523 (7559), 196-199. 
(25) Spano, F. C.; Silva, C. H- and J-Aggregate Behavior in Polymeric Semiconductors. Annu. Rev. Phys. Chem. 2014, 65, 477500.

(26) Yamagata, H.; Spano, F. C. Interplay between Intrachain and Interchain Interactions in Semiconducting Polymer Assemblies: The HJ-Aggregate Model. J. Chem. Phys. 2012, 136 (18), 184901.

(27) Niles, E. T.; Roehling, J. D.; Yamagata, H.; Wise, A. J.; Spano, F. C.; Moulé, A. J.; Grey, J. K. J-Aggregate Behavior in Poly-3Hexylthiophene Nanofibers. J. Phys. Chem. Lett. 2012, 3 (2), 259263.

(28) Watson, S. M. D.; Galindo, M. A.; Horrocks, B. R.; Houlton, A. Mechanism of Formation of Supramolecular DNA-Templated Polymer Nanowires. J. Am. Chem. Soc. 2014, 136 (18), 6649-6655.

(29) Kim, H. J.; Skinner, M.; Yu, H.; Oh, J. H.; Briseno, A. L.; Emrick, T.; Kim, B. J.; Hayward, R. C. Water Processable Polythiophene Nanowires by Photo-Cross-Linking and ClickFunctionalization. Nano Lett. 2015, 15 (9), 5689-5695.

(30) Belosludov, R. V.; Sato, H.; Farajian, A. A.; Mizuseki, H.; Kawazoe, Y. Theoretical Study of Molecular Enamel Wires Based on Polythiophene-Cyclodextrin Inclusion Complexes. Mol. Cryst. Liq. Cryst. 2003, 406, 1-10.

(31) Van Den Boogaard, M.; Bonnet, G.; Van't Hof, P.; Wang, Y.; Brochon, C.; Van Hutten, P.; Lapp, A.; Hadziioannou, G. Synthesis of Insulated Single-Chain Semiconducting Polymers Based on Polythiophene, Polyfluorene, and $\beta$-Cyclodextrin. Chem. Mater. 2004, 16 (23), 4383-4385.

(32) Shimomura, T.; Akai, T.; Fujimori, M.; Heike, S.; Hashizume, T.; Ito, K. Conductivity Measurement of Insulated Molecular Wire Formed by Molecular Nanotube and Polyaniline. Synth. Met. 2005, $153(1-3), 497-500$.

(33) Masai, H.; Terao, J. Stimuli-Responsive Functionalized Insulated Conjugated Polymers. Polym. J. 2017, 49 (12), 805-814.

(34) Li, C.; Numata, M.; Bae, A. H.; Sakurai, K.; Shinkai, S. SelfAssembly of Supramolecular Chiral Insulated Molecular Wire. J. Am. Chem. Soc. 2005, 127 (13), 4548-4549.

(35) Hollingsworth, W. R.; Segura, C.; Balderrama, J.; Lopez, N.; Schleissner, P.; Ayzner, A. L. Exciton Transfer and Emergent Excitonic States in Oppositely-Charged Conjugated Polyelectrolyte Complexes. J. Phys. Chem. B 2016, 120 (31), 7767-7774.

(36) Knaapila, M.; Costa, T.; Garamus, V. M.; Kraft, M.; Drechsler, M.; Scherf, U.; Burrows, H. D. Conjugated Polyelectrolyte (CPE) Poly\{3-[6-(N -Methylimidazolium)Hexyl]-2, 5-Thiophene\} Complexed with DNA: Relation between Colloidal Level Solution Structure and Chromic Effects. Macromolecules 2014, 47 (12), 4017-4027.

(37) Knudsen, J. B.; Liu, L.; Bank Kodal, A. L.; Madsen, M.; Li, Q.; Song, J.; Woehrstein, J. B.; Wickham, S. F. J.; Strauss, M. T.; Schueder, F.; Vinther, J.; Krissanaprasit, A.; Gudnason, D.; Smith, A. A. A.; Ogaki, R.; Zelikin, A. N.; Besenbacher, F.; Birkedal, V.; Yin, P.; Shih, W. M.; Jungmann, R.; Dong, M.; Gothelf, K. V. Routing of Individual Polymers in Designed Patterns. Nat. Nanotechnol. 2015, 10 (10), 892-898.

(38) Hsu, W. L.; Li, Y. C.; Chen, H. L.; Liou, W.; Jeng, U. S.; Lin, H. K.; Liu, W. L.; Hsu, C. S. Thermally-Induced Order-Order Transition of DNA-Cationic Surfactant Complexes. Langmuir 2006, 22, 75217527.

(39) Selmi, D. N.; Adamson, R. J.; Attrill, H.; Goddard, A. D.; Gilbert, R. J. C.; Watts, A.; Turberfield, A. J. DNA-Templated Protein Arrays for Single-Molecule Imaging. Nano Lett. 2011, 11 (2), 657660.

(40) Hou, C.; Guan, S.; Wang, R.; Zhang, W.; Meng, F.; Zhao, L.; $\mathrm{Xu}$, J.; Liu, J. Supramolecular Protein Assemblies Based on DNA Templates. J. Phys. Chem. Lett. 2017, 8 (17), 3970-3979.

(41) Li, Y.; Liu, Z.; Yu, G.; Jiang, W.; Mao, C. Self-Assembly of Molecule-like Nanoparticle Clusters Directed by DNA Nanocages. J. Am. Chem. Soc. 2015, 137 (13), 4320-4323.

(42) Wang, M.; Silva, G. L.; Armitage, B. A. DNA-Templated Formation of a Helical Cyanine Dye J-Aggregate. J. Am. Chem. Soc. 2000, 122 (41), 9977-9986.
(43) Benvin, A. L.; Creeger, Y.; Fisher, G. W.; Ballou, B.; Waggoner, A. S.; Armitage, B. A. Fluorescent DNA Nanotags: Supramolecular Fluorescent Labels Based on Intercalating Dye Arrays Assembled on Nanostructured DNA Templates. J. Am. Chem. Soc. 2007, 129 (7), 2025-2034.

(44) Janssen, P. G. A.; Vandenbergh, J.; Van Dongen, J. L. J.; Meijer, E. W.; Schenning, A. P. H. J. SsDNA Templated Self-Assembly of Chromophores. J. Am. Chem. Soc. 2007, 129 (19), 6078-6079.

(45) Björk, P.; Persson, N. K.; Peter, K.; Nilsson, R.; Åsberg, P.; Inganäs, O. Dynamics of Complex Formation between Biological and Luminescent Conjugated Polyelectrolytes - A Surface Plasmon Resonance Study. Biosens. Bioelectron. 2005, 20 (9), 1764-1771.

(46) Dubin, F.; Melet, R.; Barisien, T.; Grousson, R.; Legrand, L.; Schott, M.; Voliotis, V. Macroscopic Coherence of a Single Exciton State in an Organic Quantum Wire. Nat. Phys. 2006, 2, 32-35.

(47) Cacialli, F.; Wilson, J. S.; Michels, J. J.; Daniel, C.; Silva, C.; Friend, R. H.; Severin, N.; Samori, P.; Rabe, J. P.; O'connell, M. J.; et al. Cyclodextrin-Threaded Conjugated Polyrotaxanes as Insulated Molecular Wires with Reduced Interstrand Interactions. Nat. Mater. 2002, 1 (3), 160-164.

(48) Béra-Abérem, M.; Najari, A.; Ho, H. A.; Gravel, J. F.; Nobert, P.; Boudreau, D.; Leclerc, M. Protein Detecting Arrays Based on Cationic Polythiophene-DNA-Aptamer Complexes. Adv. Mater. 2006, 18 (20), 2703-2707.

(49) Guan, H.; Cai, M.; Chen, L.; Wang, Y.; He, Z. Label-Free DNA Sensor Based on Fluorescent Cationic Polythiophene for the Sensitive Detection of Hepatitis B Virus Oligonucleotides. Luminescence 2010, 25 (4), 311-316.

(50) Zhan, R.; Fang, Z.; Liu, B. Naked-Eye Detection and Quantification of Heparin in Serum with a Cationic Polythiophene. Anal. Chem. 2010, 82 (4), 1326-1333.

(51) Ho, H. A.; Boissinot, M.; Bergeron, M. G.; Corbeil, G.; Dore, K.; Boudreau, D.; Leclerc, M. Colorimetric and Fluorometric Detection of Nucleic Acids Using Cationic Polythiophene Derivatives. Angew. Chem., Int. Ed. 2002, 41 (9), 1548-1551.

(52) Charlebois, I.; Gravel, C.; Arrad, N.; Boissinot, M.; Bergeron, M. G.; Leclerc, M. Impact of DNA Sequence and Oligonucleotide Length on a Polythiophene-Based Fluorescent DNA Biosensor. Macromol. Biosci. 2013, 13 (6), 717-722.

(53) Rubio-Magnieto, J.; Azene, E. G.; Knoops, J.; Knippenberg, S.; Delcourt, C.; Thomas, A.; Richeter, S.; Mehdi, A.; Dubois, P.; Lazzaroni, R.; et al. Self-Assembly and Hybridization Mechanisms of DNA with Cationic Polythiophene. Soft Matter 2015, 11, 6460-6471.

(54) Kypr, J.; Kejnovská, I.; Renčiuk, D.; Vorlíčková, M. Circular Dichroism and Conformational Polymorphism of DNA. Nucleic Acids Res. 2009, 37 (6), 1713-1725.

(55) Peterhans, L.; Alloa, E.; Sheima, Y.; Vannay, L.; Leclerc, M.; Corminboeuf, C.; Hayes, S. C.; Banerii, N. Salt-Induced Thermochromism of a Conjugated Polyelectrolyte. Phys. Chem. Chem. Phys. 2017, 19 (42), 28853-28866.

(56) Banerji, N.; Cowan, S.; Vauthey, E.; Heeger, A. J. Ultrafast Relaxation of the Poly(3-Hexylthiophene) Emission Spectrum. J. Phys. Chem. C 2011, 115 (19), 9726-9739.

(57) Shaner, N. C.; Patterson, G. H.; Davidson, M. W. Advances in Fluorescent Protein Technology. J. Cell Sci. 2007, 120 (24), 42474260.

(58) Rubio-Magnieto, J.; Thomas, A.; Richeter, S.; Mehdi, A.; Dubois, P.; Lazzaroni, R.; Clement, S.; Surin, M. Chirality in DNA- $\pi$ Conjugated Polymer Supramolecular Structures: Insights into the Self-Assembly. Chem. Commun. 2013, 49 (48), 5483-5485.

(59) Digennaro, A.; Wennemers, H.; Joshi, G.; Schmid, S.; MenaOsteritz, E.; Bäuerle, P. Chiral Suprastructures of Asymmetric Oligothiophene-Hybrids Induced by a Single Proline. Chem. Commun. 2013, 49 (93), 10929-10931.

(60) Nilsson, K. P. R.; Rydberg, J.; Baltzer, L.; Inganäs, O. Twisting Macromolecular Chains: Self-Assembly of a Chiral Supermolecule from Nonchiral Polythiophene Polyanions and Random-Coil Synthetic Peptides. Proc. Natl. Acad. Sci. U. S. A. 2004, 101 (31), 11197-11202. 
(61) Ewbank, P. C.; Nuding, G.; Suenaga, H.; McCullough, R. D.; Shinkai, S. Amine Functionalized Polythiophenes: Synthesis and Formation of Chiral, Ordered Structures on DNA Substrates. Tetrahedron Lett. 2001, 42 (2), 155-157.

(62) Langeveld-Voss, B. M. W.; Christiaans, M. P. T.; Janssen, R. A. J.; Meijer, E. W. Inversion of Optical Activity of Chiral Polythiophene Aggregates by a Change of Solvent. Macromolecules 1998, 31 (19), 6702-6704.

(63) Balaz, M.; Tannir, S.; Varga, K. Chiral Multichromophoric Supramolecular Nanostructures Assembled by Single Stranded DNA and RNA Templates. Coord. Chem. Rev. 2017, 349, 66-83.

(64) Mills, J. B.; Vacano, E.; Hagerman, P. J. Flexibility of SingleStranded DNA: Use of Gapped Duplex Helices to Determine the Persistence Lengths of Poly(dT) and Poly(dA). J. Mol. Biol. 1999, 285 (1), 245-257.

(65) Konorov, S. O.; Georg Schulze, H.; Addison, C. J.; Haynes, C. A.; Blades, M. W.; Turner, R. F. B. Ultraviolet Resonance Raman Spectroscopy of Locked Single-Stranded Oligo(dA) Reveals Conformational Implications of the Locked Ribose in LNA. J. Raman Spectrosc. 2009, 40 (9), 1162-1171.

(66) Martin, T. P.; Wise, A. J.; Busby, E.; Gao, J.; Roehling, J. D.; Ford, M. J.; Larsen, D. S.; Moulé, A. J.; Grey, J. K. Packing Dependent Electronic Coupling in Single Poly(3-Hexylthiophene) H- and JAggregate Nanofibers. J. Phys. Chem. B 2013, 117 (16), 4478-4487.

(67) Gao, J.; Kamps, A.; Park, S. J.; Grey, J. K. Encapsulation of Poly(3-Hexylthiophene) J-Aggregate Nanofibers with an Amphiphilic Block Copolymer. Langmuir 2012, 28 (47), 16401-16407.

(68) Ziffer, M. E.; Jo, S. B.; Liu, Y.; Zhong, H.; Mohammed, J. C.; Harrison, J. S.; Jen, A. K. Y.; Ginger, D. S. Tuning H-and J-Aggregate Behavior in $\pi$-Conjugated Polymers via Noncovalent Interactions. J. Phys. Chem. C 2018, 122 (33), 18860-18869.

(69) Antao, V. P.; Gray, D. M. CD Spectral Comparisons of the Acid-Induced Structures of Poly $[\mathrm{d}(\mathrm{A})]$, Poly $[\mathrm{r}(\mathrm{A})]$, Poly $[\mathrm{d}(\mathrm{C})]$, and Poly[r(C)]. J. Biomol. Struct. Dyn. 1993, 10 (5), 819-839.

(70) Saxena, S.; Joshi, S.; Shankaraswamy, J.; Tyagi, S.; Kukreti, S. Magnesium and Molecular Crowding of the Cosolutes Stabilize the IMotif Structure at Physiological pH. Biopolymers 2017, 107 (7), e23018.

(71) Smith, K. C.; Hanawalt, P. C. Molecular Photobiology: Inactivation and Recovery; Academic Press: London, 1969.

(72) Davis, T. M.; McFail-Isom, L.; Keane, E.; Williams, L. D. Melting of a DNA Hairpin without Hyperchromism. Biochemistry 1998, 37 (19), 6975-6978.

(73) Fodor, S. P. A.; Rava, R. P.; Hays, T. R.; Spiro, T. G. Ultraviolet Resonance Raman-Spectroscopy Of The Nucleotides With 266-, 240-, 218-, And 200-nm Pulsed Laser Excitation. J. Am. Chem. Soc. 1985, 107 (6), 1520-1529.

(74) Wen, Z. Q.; Thomas, G. J. UV Resonance Raman Spectroscopy of DNA and Protein Constituents of Viruses: Assignments and Cross Sections for Excitations at 257, 244, 238, and $229 \mathrm{~nm}$. Biopolymers 1998, 45 (3), 247-256.

(75) Benevides, J. M.; Kang, C.; Thomas, G. J. Raman Signature of the Four-Stranded Intercalated Cytosine Motif in Crystal and Solution Structures of DNA Deoxycytidylates d(CСCT) and d(C8). Biochemistry 1996, 35 (18), 5747-5755.

(76) Wen, Z. Q.; Overman, S. A.; Thomas, G. J. Structure and Interactions of the Single-Stranded DNA Genome of Filamentous Virus Fd: Investigation by Ultraviolet Resonance Raman Spectroscopy. Biochemistry 1997, 36 (25), 7810-7820.

(77) Toyama, A.; Takino, Y.; Takeuchi, H.; Harada, I. Ultraviolet Resonance Raman Spectra of Ribosyl C(1')-Deuterated Purine Nucleosides: Evidence of Vibrational Coupling between Purine and Ribose Rings. J. Am. Chem. Soc. 1993, 115 (24), 11092-11098.

(78) Miskovsky, P.; Tomkova, A.; Chinsky, L.; Turpin, P. Y. Conformational Transitions of Poly (dI-dC) in Aqueous Solution as Studied by Ultraviolet Resonance Raman Spectroscopy. J. Biomol. Struct. Dyn. 1993, 11 (3), 655-669.

(79) Tomkova, A.; Miskovsky, P.; Chinsky, L.; Turpin, P. Y. UV Resonance Raman Contribution to Structure Determination of the $\mathrm{X}$
Form of Double-Stranded Poly(dA-dT). J. Mol. Struct. 1995, 344 (12), $11-20$.

(80) Hunter, C. A. Sequence-Dependent DNA Structure The Role of Base Stacking Interactions. J. Mol. Biol. 1993, 230, 1025-1054.

(81) Guckian, K. M.; Schweitzer, B. A.; Ren, R. X. F.; Sheils, C. J.; Tahmassebi, D. C.; Kool, E. T. Factors Contributing to Aromatic Stacking in Water: Evaluation in the Context of DNA. J. Am. Chem. Soc. 2000, 122 (10), 2213-2222.

(82) Pascoe, D. J.; Ling, K. B.; Cockroft, S. L. The Origin of Chalcogen-Bonding Interactions. J. Am. Chem. Soc. 2017, 139 (42), 15160-15167.

(83) Fossépré, M.; Trévisan, M. E.; Cyriaque, V.; Wattiez, R.; Beljonne, D.; Richeter, S.; Clément, S.; Surin, M. Detection of the Enzymatic Cleavage of DNA through Supramolecular Chiral Induction to a Cationic Polythiophene. ACS Appl. Bio Mater. 2019, 2 (5), 2125-2136.

(84) Leclercq, M.; Rubio-Magnieto, J.; Mohammed, D.; Gabriele, S.; Leclercq, L.; Cottet, H.; Richeter, S.; Clément, S.; Surin, M. Supramolecular Self-Assembly of DNA with a Cationic Polythiophene: From Polyplexes to Fibers. ChemNanoMat 2019, 5, 703-709.

(85) Tsoi, W. C.; James, D. T.; Kim, J. S.; Nicholson, P. G.; Murphy, C. E.; Bradley, D. D. C.; Nelson, J.; Kim, J. S. The Nature of In-Plane Skeleton Raman Modes of P3HT and Their Correlation to the Degree of Molecular Order in P3HT:PCBM Blend Thin Films. J. Am. Chem. Soc. 2011, 133 (25), 9834-9843.

(86) Pron, A.; Louarn, G.; Lapkowski, M.; Zagorska, M.; GlowczyZubek, J.; Lefrant, S. In Situ" Raman Spectroelectrochemical Studies of Poly(3,3'-Dibutoxy-2,2'-Bithiophene). Macromolecules 1995, 28, 4644-4649.

(87) Louarn, G.; Trznadel, M.; Buisson, J. P.; Laska, J.; Pron, A.; Lapkowski, M.; Lefrant, S. Raman Spectroscopic Studies of Regioregular Poly(3-Alkylthiophenes). J. Phys. Chem. 1996, 100 (30), 12532-12539.

(88) Myers, A. B.; Mathies, R. A.; Spiro, T. G. Biological Applications of Raman Spectroscopy: Resonance Raman Spectra of Polyenes and Aromatics; John Wiley \& Sons, Ltd., 1987; Vol. 2.

(89) Cook, S.; Furube, A.; Katoh, R. Analysis of the excited states of regioregular polythiophene P3HT. Energy Environ. Sci. 2008, 1 (2), 294-299.

(90) Guo, J.; Ohkita, H.; Benten, H.; Ito, S. Near-IR Femtosecond Transient Absorption Spectroscopy of Ultrafast Polaron and Triplet Exciton Formation in Polythiophene Films with Different Regioregularities. J. Am. Chem. Soc. 2009, 131 (46), 16869-16880.

(91) Bargigia, I.; Zucchetti, E.; Kandada, A. R. S.; Moreira, M.; Bossio, C.; Wong, W. P. D.; Miranda, P. B.; Decuzzi, P.; Soci, C.; D'Andrea, C.; et al. The Photophysics of Polythiophene Nanoparticles for Biological Applications. ChemBioChem 2019, 20 (4), 532-536.

(92) Zucchetti, E.; Zangoli, M.; Bargigia, I.; Bossio, C.; Di Maria, F.; Barbarella, G.; D'Andrea, C.; Lanzani, G.; Antognazza, M. R. Poly(3Hexylthiophene) Nanoparticles for Biophotonics: Study of the Mutual Interaction with Living Cells. J. Mater. Chem. B 2017, 5 (3), 565-574.

(93) Yang, X.; Dykstra, T. E.; Scholes, G. D. Photon-Echo Studies of Collective Absorption and Dynamic Localization of Excitation in Conjugated Polymers and Oligomers. Phys. Rev. B: Condens. Matter Mater. Phys. 2005, 71 (4), 1-15.

(94) Wells, N. P.; Boudouris, B. W.; Hillmyer, M. A.; Blank, D. A. Intramolecular Exciton Relaxation and Migration Dynamics in Poly(3-Hexylthiophene). J. Phys. Chem. C 2007, 111 (42), 1540415414.

(95) Hwang, I.; Scholes, G. D. Electronic Energy Transfer and Quantum-Coherence in $\pi$-Conjugated Polymers. Chem. Mater. 2011, 23 (3), 610-620.

(96) Tozer, O. R.; Barford, W. Exciton Dynamics in Disordered Poly(p-Phenylenevinylene). 1. Ultrafast Interconversion and Dynamical Localization. J. Phys. Chem. A 2012, 116 (42), 10310-10318.

(97) Banerji, N. Sub-Picosecond Delocalization in the Excited State of Conjugated Homopolymers and Donor-Acceptor Copolymers. J. Mater. Chem. C 2013, 1 (18), 3052-3066. 
(98) Collini, E.; Scholes, G. D. Coherent Intrachain Energy Migration in a Conjugated Polymer at Room Temperature. Science 2009, 323 (5912), 369-373.

(99) Westenhoff, S.; Beenken, W. J. D.; Friend, R. H.; Greenham, N. C.; Yartsev, A.; Sundström, V. Anomalous Energy Transfer Dynamics Due to Torsional Relaxation in a Conjugated Polymer. Phys. Rev. Lett. 2006, 97 (16), 166804.

(100) Gallaher, J. K.; Chen, K.; Huff, G. S.; Prasad, S. K. K.; Gordon, K. C.; Hodgkiss, J. M. Evolution of Nonmirror Image Fluorescence Spectra in Conjugated Polymers and Oligomers. J. Phys. Chem. Lett. 2016, 7 (17), 3307-3312.

(101) Kim, T. W.; Kim, W.; Park, K. H.; Kim, P.; Cho, J. W.; Shimizu, H.; Iyoda, M.; Kim, D. Chain-Length-Dependent Exciton Dynamics in Linear Oligothiophenes Probed Using Ensemble and Single-Molecule Spectroscopy. J. Phys. Chem. Lett. 2016, 7 (3), 452458.

(102) Okawa, Y.; Mandal, S. K.; Hu, C.; Tateyama, Y.; Goedecker, S.; Tsukamoto, S.; Hasegawa, T.; Gimzewski, J. K.; Aono, M. Chemical Wiring and Soldering toward All-Molecule Electronic Circuitry. J. Am. Chem. Soc. 2011, 133 (21), 8227-8233.

(103) Yamagata, H.; Spano, F. C. Vibronic Coupling in Quantum Wires: Applications to Polydiacetylene. J. Chem. Phys. 2011, 135 (5), 054906.

(104) Holcman, J.; Al Choueiry, A.; Enderlin, A.; Hameau, S.; Barisien, T.; Legrand, L. Coherent Control of the Optical Emission in a Single Organic Quantum Wire. Nano Lett. 2011, 11 (10), 44964502 .

(105) Dore, K.; Dubus, S.; Ho, H. A.; Levesque, I.; Brunette, M.; Corbeil, G.; Boissinot, M.; Boivin, G.; Bergeron, M. G.; Boudreau, D.; et al. Fluorescent Polymeric Transducer for the Rapid, Simple, and Specific Detection of Nucleic Acids at the Zeptomole Level. J. Am. Chem. Soc. 2004, 126 (13), 4240-4244.

(106) Dans, P. D.; Ivani, I.; Hospital, A.; Portella, G.; González, C.; Orozco, M. How Accurate Are Accurate Force-Fields for B-DNA? Nucleic Acids Res. 2017, 45 (7), 4217-4230.

(107) Ivani, I.; Dans, P. D.; Noy, A.; Pérez, A.; Faustino, I.; Hospital, A.; Walther, J.; Andrio, P.; Goñi, R.; Balaceanu, A.; et al. Parmbsc1: A Refined Force Field for DNA Simulations. Nat. Methods 2016, 13 (1), 55-58.

(108) Jorgensen, W. L.; Chandrasekhar, J.; Madura, J. D.; Impey, R. W.; Klein, M. L. Comparison of Simple Potential Functions for Simulating Liquid Water. J. Chem. Phys. 1983, 79, 926-935.

(109) Joung, I. S.; Cheatham, T. E., 3rd Determination of Alkali and Halide Monovalent Ion Parameters for Use in Explicitly Solvated Biomolecular Simulations. J. Phys. Chem. B 2008, 112 (30), 90209041.

(110) Frisch, M. J.; Trucks, G. W.; Schlegel, H. B.; Scuseria, G. E.; Robb, M. A.; Cheeseman, J. R.; Scalmani, G.; Barone, V.; Mennucci, B.; Petersson, G. A.; et al. Gaussian 09, Revision A.1; Gaussian, Inc.: Wallingford, CT, 2009.

(111) Tomasi, J.; Cammi, R.; Mennucci, B.; Cappelli, C.; Corni, S. Molecular Properties in Solution Described with a Continuum Solvation Model. Phys. Chem. Chem. Phys. 2002, 4 (23), 5697-5712.

(112) Amovilli, C.; Barone, V.; Cammi, R.; Cancès, E.; Cossi, M.; Mennucci, B.; Pomelli, C. S.; Tomasi, J. Recent Advances in the Description of Solvent Effects with the Polarizable Continuum Model. In Adv. Quantum Chem.; Löwdin, P. O., Ed.; Academic Press, 1998; Vol. 32, pp 227-261.

(113) Kohn, W.; Sham, L. J. Self-Consistent Equations Including Exchange and Correlation Effects. Phys. Rev. 1965, 140 (4A), A1133A1138.

(114) Burke, K.; Wagner, L. O. DFT in a Nutshell. Int. J. Quantum Chem. 2013, 113 (2), 96-101.

(115) Becke, A. D. Density-functional Thermochemistry. III. The Role of Exact Exchange. J. Chem. Phys. 1993, 98 (7), 5648-5652.

(116) Lee, C.; Yang, W.; Parr, R. G. Development of the ColleSalvetti Correlation-Energy Formula into a Functional of the Electron Density. Phys. Rev. B: Condens. Matter Mater. Phys. 1988, 37 (2), 785-789.
(117) Petersson, G. A.; Al-Laham, M. A. A Complete Basis Set Model Chemistry. II. Open-shell Systems and the Total Energies of the First-row Atoms. J. Chem. Phys. 1991, 94 (9), 6081-6090.

(118) Petersson, G. A.; Bennett, A.; Tensfeldt, T. G.; Al-Laham, M. A.; Shirley, W. A.; Mantzaris, J. A Complete Basis Set Model Chemistry. I. The Total Energies of Closed-shell Atoms and Hydrides of the First-row Elements. J. Chem. Phys. 1988, 89 (4), 2193-2218.

(119) Qiu, M.; Long, S.; Li, B.; Yan, L.; Xie, W.; Niu, Y.; Wang, X.; Guo, Q.; Xia, A. Toward an Understanding of How the Optical Property of Water-Soluble Cationic Polythiophene Derivative Is Altered by the Addition of Salts: The Hofmeister Effect. J. Phys. Chem. C 2013, 117 (42), 21870-21878.

(120) Wang, J.; Wolf, R. M.; Caldwell, J. W.; Kollman, P. A.; Case, D. A. Development and Testing of a General Amber Force Field. J. Comput. Chem. 2004, 25 (9), 1157-1174.

(121) GAFF2 is a public domain force field, an upgrade of the previously released general AMBER force field (GAFF). It is available with the distribution of AmberTools17 and can be downloaded from http://ambermd.org. A publication for it is currently under preparation.

(122) Le Grand, S.; Götz, A. W.; Walker, R. C. SPFP: Speed without Compromise-A Mixed Precision Model for GPU Accelerated Molecular Dynamics Simulations. Comput. Phys. Commun. 2013, 184 (2), 374-380. 